\title{
Generalization of Fuzzy Laplace Transforms of Fuzzy Fractional Derivatives about the General Fractional Order $n-1<\beta<n$
}

\author{
Amal Khalaf Haydar and Ruaa Hameed Hassan \\ Department of Mathematics, Faculty of Education for Girls, Kufa University, Najaf, Iraq \\ Correspondence should be addressed to Amal Khalaf Haydar; amalkh.hayder@uokufa.edu.iq
}

Received 17 July 2015; Accepted 13 October 2015

Academic Editor: Zhen-Lai Han

Copyright (c) 2016 A. K. Haydar and R. H. Hassan. This is an open access article distributed under the Creative Commons Attribution License, which permits unrestricted use, distribution, and reproduction in any medium, provided the original work is properly cited.

\begin{abstract}
The main aim in this paper is to use all the possible arrangements of objects such that $r_{1}$ of them are equal to 1 and $r_{2}$ (the others) of them are equal to 2, in order to generalize the definitions of Riemann-Liouville and Caputo fractional derivatives (about order $0<\beta<n)$ for a fuzzy-valued function. Also, we find fuzzy Laplace transforms for Riemann-Liouville and Caputo fractional derivatives about the general fractional order $n-1<\beta<n$ under H-differentiability. Some fuzzy fractional initial value problems (FFIVPs) are solved using the above two generalizations.
\end{abstract}

\section{Introduction}

Fuzzy Fractional Differential Equations (FFDEs) can offer a more comprehensive account of the process or phenomenon. This has recently captured much attention in FFDEs. As the derivative of a function is defined in the sense of RiemannLiouville, Grünwald-Letnikov, or Caputo in fractional calculus, the used derivative is to be specified and defined in FFDEs as well [1].

Many researchers have worked on the field of Fuzzy Fractional Differential Equations (FFDEs); for example, Salahshour et al. [2] dealt with the solutions of FFDEs under Riemann-Liouville H-differentiability by fuzzy Laplace transforms; Mazandarani and Kamyad [1] presented the solution to FFIVP under Caputo-type fuzzy fractional derivatives by a modified fractional Euler method; Wu and Baleanu [3] proposed a novel modification of the variational iteration method (VIM) by means of the Laplace transform; they extended the method successfully to fractional differential equations; Ahmadian et al. [4] reveal a computational method based on using a Tau method with Jacobi polynomials for the solution of fuzzy linear fractional differential equations of order $0<v<1$, and Allahviranloo et al. [5] introduced the fuzzy Caputo fractional differential equations under the generalized Hukuhara differentiability.
This paper is arranged as follows. Basic concepts are given in Section 2. In Section 3, the general formula of the fuzzy Riemann-Liouville fractional derivatives and the general formula of Laplace transforms of the fuzzy RiemannLiouville fractional derivatives for a fuzzy-valued function $f$ are found. In Section 4, the general formula of the fuzzy Caputo fractional derivatives and the general formula of Laplace transforms of the fuzzy Caputo fractional derivatives for a fuzzy-valued function $f$ are found. In Section 5 , conclusions are drawn.

\section{Basic Concepts}

In this section, we give the basic concepts which are needed in the next sections. We denote $C^{F}[a, b]$ as the space of all continuous fuzzy-valued functions on $[a, b]$. Also, we denote the space of all Lebesgue integrable fuzzy-valued functions on the bounded interval $[a, b] \subset \mathbb{R}$ by $L^{F}[a, b]$.

Theorem 1 (see [6]). Let $n$ be a positive integer. Let $D^{n-1} f$ be continuous on $J=[0, \infty) ; C$ is the class of piecewise continuous functions on $J^{\prime}=(0, \infty)$ and integrable on any finite subinterval of $J=[0, \infty)$ and let $v>0$. Then, one finds the following: 
(a) If $D^{n} f$ is of class $C$, then

$$
D^{-v} f(x)=D^{-v-n}\left[D^{n} f(x)\right]+Q_{n}(x, v)
$$

and

(b) if $D^{n} f$ is continuous on $J$, then for $x>0$

$$
D^{n}\left[D^{-v} f(x)\right]=D^{-v}\left[D^{n} f(x)\right]+Q_{n}(x, v-n),
$$

where

$$
Q_{n}(x, v)=\sum_{k=0}^{n-1} \frac{x^{v+k}}{\Gamma(v+k+1)} D^{k} f(0) .
$$

Definition 2 (see [7]). A fuzzy number $u$ in parametric form is a pair $(\underline{u}, \bar{u})$ of functions $\underline{u}(r), \bar{u}(r), 0 \leq r \leq 1$, which satisfy the following requirements.

(1) $\underline{u}(r)$ is a bounded nondecreasing left continuous function in $(0,1]$ and right continuous at 0 .

(2) $\bar{u}(r)$ is a bounded nonincreasing left continuous function in $(0,1]$ and right continuous at 0 .

(3) Consider $\underline{u}(r) \leq \bar{u}(r), 0 \leq r \leq 1$.

We denote the set of all real numbers by $\mathbb{R}$ and the set of all fuzzy numbers on $\mathbb{R}$ is indicated by $E$.

Definition 3 (see [8]). Let $x, y \in E$. If there exists $z \in E$ such that $x=y+z$, then $z$ is called the H-difference of $x$ and $y$, and it is denoted by $x \ominus y$. The sign " $\ominus$ " always stands for $\mathrm{H}$-difference and also note that $x \ominus y \neq x+(-1) y$.

Definition 4 (see [9]). Let $f(x)$ be continuous fuzzyvalued function; suppose that $f(x) e^{-s x}$ is improper fuzzy Rimann-integrable on $[0, \infty)$ then $\int_{0}^{\infty} f(x) e^{-s x} d x$ is called fuzzy Laplace transforms and is denoted as $L[f(x)]=$ $\int_{0}^{\infty} f(x) e^{-s x} d x,(s>0)$.

We have

$$
\begin{aligned}
& \int_{0}^{\infty} f(x) e^{-s x} d x \\
& \quad=\left(\int_{0}^{\infty} \underline{f}(x ; r) e^{-s x} d x, \int_{0}^{\infty} \bar{f}(x ; r) e^{-s x} d x\right) ;
\end{aligned}
$$

also by using the definition of classical Laplace transform:

$$
\begin{aligned}
& \ell[\underline{f}(x ; r)]=\int_{0}^{\infty} \underline{f}(x ; r) e^{-s x} d x, \\
& \ell[\bar{f}(x ; r)]=\int_{0}^{\infty} \bar{f}(x ; r) e^{-s x} d x
\end{aligned}
$$

then, we follow:

$$
L[f(x)]=(\ell[\underline{f}(x ; r)], \ell[\bar{f}(x ; r)]) .
$$

Definition 5 (see [2]). Let $f \in C^{F}[a, b] \cap L^{F}[a, b]$. The fuzzy Riemann-Liouville integral of fuzzy-valued function $f$ is defined as follows:

$$
\left(I_{a+}^{\beta} f\right)(x)=\frac{1}{\Gamma(\beta)} \int_{a}^{x} \frac{f(t) d t}{(x-t)^{1-\beta}}, \quad x>a, 0<\beta \leq 1 .
$$

\section{Generalization of Fuzzy Laplace Transforms of the Fuzzy Riemann-Liouville Fractional Derivatives of Order $n-1<\beta<n$}

In this section, we define Riemann-Liouville fractional derivatives of the general fractional order $0<\beta<n$ and we find fuzzy Laplace transforms for Riemann-Liouville fractional derivatives of the general fractional order $n-1<\beta<n$ for fuzzy-valued function $f$ under H-differentiability.

Definition 6. Let $f(x) \in C^{F}[0, b] \cap L^{F}[0, b]$, and $\lceil\beta\rceil$ and $\lfloor\beta\rfloor$ are values of $\beta$ rounded up and down to the nearest integer number, respectively. One can see that $\phi(x)=(1 / \Gamma(\lceil\beta\rceil-$ $\beta)) \int_{0}^{x}\left(f(t) d t /(x-t)^{1-\lceil\beta\rceil+\beta}\right)$, and the functions $\phi_{i_{1}, i_{2}, \ldots, i_{j}, 1}$ and $\phi_{i_{1}, i_{2}, \ldots, i_{j}, 2}$ are defined as:

$$
\begin{gathered}
\phi_{i_{1}, i_{2}, \ldots, i_{j}, 1}\left(x_{0}\right)=\lim _{h \rightarrow 0^{+}} \frac{\phi_{i_{1}, i_{2}, \ldots, i_{j}}\left(x_{0}+h\right) \ominus \phi_{i_{1}, i_{2}, \ldots, i_{j}}\left(x_{0}\right)}{h} \\
=\lim _{h \rightarrow 0^{+}} \frac{\phi_{i_{1}, i_{2}, \ldots, i_{j}}\left(x_{0}\right) \ominus \phi_{i_{1}, i_{2}, \ldots, i_{j}}\left(x_{0}-h\right)}{h}, \\
\phi_{i_{1}, i_{2}, \ldots, i_{j}, 2}\left(x_{0}\right)=\lim _{h \rightarrow 0^{+}} \frac{\phi_{i_{1}, i_{2}, \ldots, i_{j}}\left(x_{0}\right) \ominus \phi_{i_{1}, i_{2}, \ldots, i_{j}}\left(x_{0}+h\right)}{-h} \\
=\lim _{h \rightarrow 0^{+}} \frac{\phi_{i_{1}, i_{2}, \ldots, i_{j}}\left(x_{0}-h\right) \ominus \phi_{i_{1}, i_{2}, \ldots, i_{j}}\left(x_{0}\right)}{-h},
\end{gathered}
$$

for $j=0,1, \ldots, n-2$, such that $i_{1}, i_{2}, \ldots, i_{j}$ are all the possible arrangements of $j$ objects which have the number given in the rule:

$$
j P_{r_{1}, r_{2}}=\frac{j !}{r_{1} ! r_{2} !}, \quad r_{1}+r_{2}=j
$$

where $r_{1}$ of them equal 1 (meaning Riemann-Liouville type derivative in the first form) and $r_{2}$ of them equal 2 (meaning Riemann-Liouville type derivative in the second form) and $\phi_{i_{1}, \ldots, i_{0}}=\phi . f(x)$ is the Riemann-Liouville type fuzzy fractional differentiable function of order $0<\beta<n, \beta \neq$ $1,2, \ldots, n-1$, at $x_{0} \in(0, b)$, if there exists an element $\left({ }^{\mathrm{RL}} D^{\beta} f\right)\left(x_{0}\right) \in C^{F}$ such that for all $0 \leq r \leq 1$ and for $h>0$ sufficiently near zero. Then:

(1) If $i_{\lceil\beta\rceil}=1$, then

$$
\begin{aligned}
& \left({ }^{\mathrm{RL}} D^{\beta} f\right)\left(x_{0}\right) \\
& \quad=\lim _{h \rightarrow 0^{+}} \frac{\phi_{i_{1}, i_{2}, \ldots, i_{[\beta]}}\left(x_{0}+h\right) \ominus \phi_{i_{1}, i_{2}, \ldots, i_{[\beta]}}\left(x_{0}\right)}{h} \\
& \quad=\lim _{h \rightarrow 0^{+}} \frac{\phi_{i_{1}, i_{2}, \ldots, i_{[\beta]}}\left(x_{0}\right) \ominus \phi_{i_{1}, i_{2}, \ldots, i_{[\beta]}}\left(x_{0}-h\right)}{h} .
\end{aligned}
$$

(2) If $i_{\lceil\beta\rceil}=2$, then

$\left({ }^{\mathrm{RL}} D^{\beta} f\right)\left(x_{0}\right)$ 


$$
\begin{aligned}
& =\lim _{h \rightarrow 0^{+}} \frac{\phi_{i_{1}, i_{2}, \ldots, i_{\lfloor\beta\rfloor}}\left(x_{0}\right) \ominus \phi_{i_{1}, i_{2}, \ldots, i_{\lfloor\beta\rfloor}}\left(x_{0}+h\right)}{-h} \\
& =\lim _{h \rightarrow 0^{+}} \frac{\phi_{i_{1}, i_{2}, \ldots, i_{\lfloor\beta\rfloor}}\left(x_{0}-h\right) \ominus \phi_{i_{1}, i_{2}, \ldots, i_{\lfloor\beta\rfloor}}\left(x_{0}\right)}{-h},
\end{aligned}
$$

for $k-1<\beta<k, k=1,2, \ldots, n$, such that $i_{1}, i_{2}, \ldots, i_{\lfloor\beta\rfloor}$ are all the possible arrangements of $\lfloor\beta\rfloor$ objects which have the number given by the rule:

$$
\lfloor\beta\rfloor P_{r_{1}, r_{2}}=\frac{\lfloor\beta\rfloor !}{r_{1} ! r_{2} !}, \quad r_{1}+r_{2}=\lfloor\beta\rfloor .
$$

If the fuzzy-valued function $f(x)$ is differentiable as in Definition 6 cases defined in (11), it is the RiemannLiouville type differentiable in the first form and denoted by $\left({ }^{\mathrm{RL}} D_{i_{1}, i_{2}, \ldots, i_{\lfloor\beta]}, 1}^{\beta} f\right)\left(x_{0}\right)$. If $f(x)$ is differentiable as in Definition 6 cases defined in (12), it is the Riemann-Liouville type differentiable in the second form and denoted by $\left({ }^{\mathrm{RL}} D_{i_{1}, i_{2}, \ldots, i_{[\beta]}, 2}^{\beta} f\right)\left(x_{0}\right)$.

We note that if we take $n=1(0<\beta<1)$ in Definition 6 we get Definition 3.2 [2] which is introduced by Salahshour et al.

Theorem 7. Let $f(x) \in C^{F}[0, b] \cap L^{F}[0, b]$ be a fuzzy-valued function such that $f(x)=[f(x ; r), \bar{f}(x ; r)]$ for $r \in[0,1], x_{0} \in$ $(0, b)$, and $\phi(x)=(1 / \Gamma(\lceil\beta\rceil-\beta)) \int_{0}^{x}\left(f(t) d t /(x-t)^{1-\lceil\beta\rceil+\beta}\right)$. Suppose that $0<\beta<n$ and $m$ is the number of repetitions of number 2 among $i_{1}, i_{2}, \ldots, i_{\lceil\beta\rceil}$ for $k-1<\beta<k, k=$ $1,2, \ldots, n$, say, $i_{k_{1}}, i_{k_{2}}, \ldots, i_{k_{m}}$, such that $k_{1}<k_{2}<\cdots<k_{m}$; that is, $i_{k_{1}}=i_{k_{2}}=\cdots=i_{k_{m}}=2$ and $0 \leq m \leq\lceil\beta\rceil$. Then, one has the following:

If $m$ is an even number, then

$$
\begin{aligned}
& \left({ }^{R L} D_{i_{1}, i_{2}, \ldots, i_{[\beta]}}^{\beta} f\right)\left(x_{0}\right) \\
& \quad=\left[\left({ }^{R L} D^{\beta} \underline{f}\right)\left(x_{0} ; r\right),\left({ }^{R L} D^{\beta} \bar{f}\right)\left(x_{0} ; r\right)\right] .
\end{aligned}
$$

If $m$ is an odd number, then

$$
\begin{aligned}
& \left({ }^{R L} D_{i_{1}, i_{2}, \ldots, i_{[\beta]}}^{\beta} f\right)\left(x_{0}\right) \\
& \quad=\left[\left({ }^{R L} D^{\beta} \bar{f}\right)\left(x_{0} ; r\right),\left({ }^{R L} D^{\beta} \underline{f}\right)\left(x_{0} ; r\right)\right],
\end{aligned}
$$

where

$$
\begin{aligned}
& \left({ }^{R L} D^{\beta} \underline{f}\right)\left(x_{0} ; r\right) \\
& =\left[\frac{1}{\Gamma(\lceil\beta\rceil-\beta)}\left(\frac{d}{d x}\right)^{\lceil\beta\rceil} \int_{0}^{x} \frac{\underline{f}(t ; r)}{(x-t)^{1-\lceil\beta\rceil+\beta}} d t\right]_{x=x_{0}}, \\
& \left({ }^{R L} D^{\beta} \bar{f}\right)\left(x_{0} ; r\right) \\
& =\left[\frac{1}{\Gamma(\lceil\beta\rceil-\beta)}\left(\frac{d}{d x}\right)^{\lceil\beta\rceil} \int_{0}^{x} \frac{\bar{f}(t ; r)}{(x-t)^{1-\lceil\beta\rceil+\beta}} d t\right]_{x=x_{0}} .
\end{aligned}
$$

Proof. Suppose that $m$ is an even number, and then $m=2 s$, $s \in N$. Now, we have two probabilities as follows.

The first probability is $\left({ }^{\mathrm{RL}} D_{i_{1}, \ldots, i_{k_{1}}, \ldots, i_{k_{2}}, \ldots, i_{k_{2 s}}, \ldots, i_{\lfloor\beta]}}^{\beta} f\right)\left(x_{0}\right)$ is the Riemann-Liouville type fuzzy fractional differentiable function in the first form $\left(i_{\lceil\beta\rceil}=1\right)$, and then from (11) of Definition 6, we have:

$$
\begin{aligned}
& \phi_{i_{1}, \ldots, i_{\lfloor\beta\rfloor}}\left(x_{0}+h\right) \ominus \phi_{i_{1}, \ldots, i_{\lfloor\beta\rfloor}}\left(x_{0}\right)=\left[\underline{\phi}_{i_{1}, \ldots, i_{\lfloor\beta\rfloor}}\left(x_{0}+h ; r\right)\right. \\
& -\underline{\phi}_{i_{1}, \ldots, i_{\lfloor\beta\rfloor}}\left(x_{0} ; r\right), \bar{\phi}_{i_{1}, \ldots, i_{\lfloor\beta\rfloor}}\left(x_{0}+h ; r\right) \\
& \left.-\bar{\phi}_{i_{1}, \ldots, i_{\lfloor\beta\rfloor}}\left(x_{0} ; r\right)\right], \\
& \phi_{i_{1}, \ldots, i_{[\beta\rfloor}}\left(x_{0}\right) \ominus \phi_{i_{1}, \ldots, i_{\lfloor\beta\rfloor}}\left(x_{0}-h\right)=\left[\underline{\phi}_{i_{1}, \ldots, i_{\lfloor\beta\rfloor}}\left(x_{0} ; r\right)\right. \\
& -\underline{\phi}_{i_{1}, \ldots, i_{\lfloor\beta\rfloor}}\left(x_{0}-h ; r\right), \bar{\phi}_{i_{1}, \ldots, i_{\lfloor\beta\rfloor}}\left(x_{0} ; r\right) \\
& \left.-\bar{\phi}_{i_{1}, \ldots, i_{\lfloor\beta\rfloor}}\left(x_{0}-h ; r\right)\right] .
\end{aligned}
$$

Multiplying both sides by $1 / h, h>0$, we obtain:

$$
\begin{aligned}
& \frac{\phi_{i_{1}, \ldots, i_{\lfloor\beta]}}\left(x_{0}+h\right) \ominus \phi_{i_{1}, \ldots, i_{\lfloor\beta\rfloor}}\left(x_{0}\right)}{h} \\
& =\left[\frac{\phi_{i_{1}, \ldots, i_{\lfloor\beta\rfloor}}\left(x_{0}+h ; r\right)-\underline{\phi}_{i_{1}, \ldots, i_{\lfloor\beta\rfloor}}\left(x_{0} ; r\right)}{h},\right. \\
& \left.\frac{\bar{\phi}_{i_{1}, \ldots, i_{l \beta]}}\left(x_{0}+h ; r\right)-\bar{\phi}_{i_{1}, \ldots, i_{[\beta]}}\left(x_{0} ; r\right)}{h}\right] \text {, } \\
& \frac{\phi_{i_{1}, \ldots, i_{\lfloor\beta\rfloor}}\left(x_{0}\right) \ominus \phi_{i_{1}, \ldots, i_{\lfloor\beta\rfloor}}\left(x_{0}-h\right)}{h} \\
& =\left[\frac{\underline{\phi}_{i_{1}, \ldots, i_{\lfloor\beta\rfloor}}\left(x_{0} ; r\right)-\underline{\phi}_{i_{1}, \ldots, i_{\lfloor\beta\rfloor}}\left(x_{0}-h ; r\right)}{h},\right. \\
& \left.\frac{\bar{\phi}_{i_{1}, \ldots, i_{\lfloor\beta\rfloor}}\left(x_{0} ; r\right)-\bar{\phi}_{i_{1}, \ldots, i_{\lfloor\beta\rfloor}}\left(x_{0}-h ; r\right)}{h}\right] \text {. }
\end{aligned}
$$

By taking $h \rightarrow 0^{+}$on both sides of the above equations, we get:

$$
\begin{aligned}
& \left({ }^{\mathrm{RL}} D^{\beta} f\right)\left(x_{0}\right) \\
& \quad=\left[\frac{d}{d x} \underline{\phi}_{i_{1}, \ldots, i_{[\beta]}}\left(x_{0} ; r\right), \frac{d}{d x} \bar{\phi}_{i_{1}, \ldots, i_{\lfloor\beta]}}\left(x_{0} ; r\right)\right] .
\end{aligned}
$$

Now, since $\phi_{i_{1}, \ldots, i_{k_{1}-1}}\left(x_{0}\right)$ is equal to the limits defined in (8) of Definition 6 , then by applying $(8)$ for $\left(k_{1}-1\right)$ times, we get

$$
\phi_{i_{1}, \ldots, i_{k_{1}-1}}\left(x_{0}\right)=\left[\underline{\phi}^{\left(k_{1}-1\right)}\left(x_{0} ; r\right), \bar{\phi}^{\left(k_{1}-1\right)}\left(x_{0} ; r\right)\right] \text {. }
$$


Since $\phi_{i_{1}, \ldots, i_{k_{1}}}\left(x_{0}\right)$ is equal to the limits defined in (9) of Definition 6, then by applying (9) once, we get:

$$
\phi_{i_{1}, \ldots, i_{k_{1}}}\left(x_{0}\right)=\left[\bar{\phi}^{\left(k_{1}\right)}\left(x_{0} ; r\right), \underline{\phi}^{\left(k_{1}\right)}\left(x_{0} ; r\right)\right] \text {. }
$$

Since $\phi_{i_{1}, \ldots, i_{k_{2}-1}}\left(x_{0}\right)$ is equal to the limits defined in (8) of Definition 6 , then by applying (8) for $\left(k_{2}-1-k_{1}\right)$ times, we get:

$$
\phi_{i_{1}, \ldots, i_{k_{2}-1}}\left(x_{0}\right)=\left[\bar{\phi}^{\left(k_{2}-1\right)}\left(x_{0} ; r\right), \underline{\phi}^{\left(k_{2}-1\right)}\left(x_{0} ; r\right)\right] .
$$

Since $\phi_{i_{1}, \ldots, i_{k_{2}}}\left(x_{0}\right)$ is equal to the limits defined in (9) of Definition 6 , then by applying (9) once, we get:

$$
\phi_{i_{1}, \ldots, i_{k_{2}}}\left(x_{0}\right)=\left[\underline{\phi}^{\left(k_{2}\right)}\left(x_{0} ; r\right), \bar{\phi}^{\left(k_{2}\right)}\left(x_{0} ; r\right)\right] \text {. }
$$

In other words, from (23) we note that, after applying (8) and (9) for any even number from $i_{k_{1}}, i_{k_{2}}, \ldots, i_{k_{m}}$, we will get an equation similar to (23). Therefore, for $\phi_{i_{1}, \ldots, i_{k_{2 s}}}\left(x_{0}\right)$, we have:

$$
\phi_{i_{1}, \ldots, i_{k_{2 s}}}\left(x_{0}\right)=\left[\underline{\phi}^{\left(k_{2 s}\right)}\left(x_{0} ; r\right), \bar{\phi}^{\left(k_{2 s}\right)}\left(x_{0} ; r\right)\right]
$$

since $2 s$ is an even number.

Finally, since $\phi_{i_{1}, \ldots, i_{|\beta|}}\left(x_{0}\right)$ is equal to the limits defined in (8) of Definition 6, then by applying (8) for $\left(\lfloor\beta\rfloor-k_{2 s}\right)$ times, we get:

$$
\phi_{i_{1}, \ldots, i_{\lfloor\beta\rfloor}}\left(x_{0}\right)=\left[\underline{\phi}^{(\lfloor\beta\rfloor)}\left(x_{0} ; r\right), \bar{\phi}^{(\lfloor\beta\rfloor)}\left(x_{0} ; r\right)\right] .
$$

Then,

$$
\begin{aligned}
& \underline{\phi}_{i_{1}, \ldots, i_{\lfloor\beta\rfloor}}\left(x_{0} ; r\right)=\underline{\phi}^{(\lfloor\beta\rfloor)}\left(x_{0} ; r\right), \\
& \bar{\phi}_{i_{1}, \ldots, i_{\lfloor\beta\rfloor}}\left(x_{0} ; r\right)=\bar{\phi}^{(\lfloor\beta\rfloor)}\left(x_{0} ; r\right) .
\end{aligned}
$$

Substituting (26) in (19) yields the following:

$$
\begin{aligned}
& \left({ }^{\mathrm{RL}} D^{\beta} f\right)\left(x_{0}\right) \\
& \quad=\left[\left(\frac{d}{d x}\right)^{\lceil\beta\rceil} \underline{\phi}\left(x_{0} ; r\right),\left(\frac{d}{d x}\right)^{\lceil\beta\rceil} \bar{\phi}\left(x_{0} ; r\right)\right] \\
& =\left[\left({ }^{\mathrm{RL}} D^{\beta} \underline{f}\right)\left(x_{0} ; r\right),\left({ }^{\mathrm{RL}} D^{\beta} \bar{f}\right)\left(x_{0} ; r\right)\right] .
\end{aligned}
$$

The second probability is $\left({ }^{\mathrm{RL}} D_{i_{1}, \ldots, i_{k_{1}}, \ldots, i_{k_{2}}, \ldots, i_{k_{2 s-1}}, \ldots i_{[\beta]}}^{\beta} f\right)\left(x_{0}\right)$ is the Riemann-Liouville type fuzzy fractional differentiable function in the second form $\left(i_{\lceil\beta\rceil}=2\right)$, and then, by applying (12) of Definition 6, we can get

$$
\begin{aligned}
& \left({ }^{\mathrm{RL}} D^{\beta} f\right)\left(x_{0}\right) \\
& \quad=\left[\frac{d}{d x} \bar{\phi}_{i_{1}, \ldots, i_{\lfloor\beta\rfloor}}\left(x_{0} ; r\right), \frac{d}{d x} \underline{\phi}_{i_{1}, \ldots, i_{\lfloor\beta\rfloor}}\left(x_{0} ; r\right)\right] .
\end{aligned}
$$

Since $2 s-2$ is an even number, then by replacing $2 s$ by $2 s-2$ in (24), we get:

$$
\phi_{i_{1}, \ldots, i_{k_{2 s-2}}}\left(x_{0}\right)=\left[\underline{\phi}^{\left(k_{2 s-2}\right)}\left(x_{0} ; r\right), \bar{\phi}^{\left(k_{2 s-2}\right)}\left(x_{0} ; r\right)\right] .
$$

Similarly, by applying (8) $\left(k_{2 s-1}-1-k_{2 s-2}\right)$ times for $\phi_{i_{1}, \ldots, i_{k_{2 s-1}-1}}\left(x_{0}\right)$ and (9) once for $\phi_{i_{1}, \ldots, i_{k_{2 s-1}}}\left(x_{0}\right)$, we get:

$$
\phi_{i_{1}, \ldots, i_{k_{2 s-1}}}\left(x_{0}\right)=\left[\bar{\phi}^{\left(k_{2 s-1}\right)}\left(x_{0} ; r\right), \underline{\phi}^{\left(k_{2 s-1}\right)}\left(x_{0} ; r\right)\right] .
$$

Finally, since $\phi_{i_{1}, \ldots, i_{\lfloor\beta]}}\left(x_{0}\right)$ is equal to the limits defined in (8) of Definition 6, then, by applying (8) for $\left(\lfloor\beta\rfloor-k_{2 s-1}\right)$ times, we get:

$$
\phi_{i_{1}, \ldots, i_{\lfloor\beta\rfloor}}\left(x_{0}\right)=\left[\bar{\phi}^{(\lfloor\beta\rfloor)}\left(x_{0} ; r\right), \underline{\phi}^{(\lfloor\beta\rfloor)}\left(x_{0} ; r\right)\right] .
$$

Then,

$$
\begin{aligned}
& \underline{\phi}_{i_{1}, \ldots, i_{\lfloor\beta\rfloor}}\left(x_{0} ; r\right)=\bar{\phi}^{(\lfloor\beta\rfloor)}\left(x_{0} ; r\right), \\
& \bar{\phi}_{i_{1}, \ldots, i_{\lfloor\beta\rfloor}}\left(x_{0} ; r\right)=\underline{\phi}^{(\lfloor\beta\rfloor)}\left(x_{0} ; r\right) .
\end{aligned}
$$

Substituting (32) in (28) yields:

$$
\begin{aligned}
& \left({ }^{\mathrm{RL}} D^{\beta} f\right)\left(x_{0}\right) \\
& \quad=\left[\left(\frac{d}{d x}\right)^{\lceil\beta\rceil} \underline{\phi}\left(x_{0} ; r\right),\left(\frac{d}{d x}\right)^{\lceil\beta\rceil} \bar{\phi}\left(x_{0} ; r\right)\right] \\
& \quad=\left[\left({ }^{\mathrm{RL}} D^{\beta} \underline{f}\right)\left(x_{0} ; r\right),\left({ }^{\mathrm{RL}} D^{\beta} \bar{f}\right)\left(x_{0} ; r\right)\right] .
\end{aligned}
$$

If $m$ is an odd number, the proof is similar.

We note that if we take $n=1(0<\beta<1)$ in Theorem 7 , we get Theorem 3.2 [2] which is found by Salahshour et al.

Corollary 8. Let $f(x) \in C^{F}[0, b] \cap L^{F}[0, b]$ be a fuzzy-valued function and $f(x)=[f(x ; r), \bar{f}(x ; r)]$ for $r \in[0,1]$ and $x_{0} \in$ $(0, b)$. Suppose that $0^{-}<\beta<3$ and $m$ is the number of repetitions of number 2 among $i_{1}, i_{2}, \ldots, i_{\lceil\beta\rceil}$ for $k-1<\beta<$ $k, k=1,2,3$. Then, one has the following.

If $f(x)$ is ${ }^{R L}[(i)-\beta]$-differentiable fuzzy-valued function, then for $0<\beta<1$

$$
\begin{aligned}
& \left({ }^{R L} D_{1}^{\beta} f\right)\left(x_{0}\right) \\
& \quad=\left[\left({ }^{R L} D^{\beta} \underline{f}\right)\left(x_{0} ; r\right),\left({ }^{R L} D^{\beta} \bar{f}\right)\left(x_{0} ; r\right)\right] .
\end{aligned}
$$

If $f(x)$ is ${ }^{R L}[(i i)-\beta]$-differentiable fuzzy-valued function, then for $0<\beta<1$

$$
\begin{aligned}
& \left({ }^{R L} D_{2}^{\beta} f\right)\left(x_{0}\right) \\
& \quad=\left[\left({ }^{R L} D^{\beta} \bar{f}\right)\left(x_{0} ; r\right),\left({ }^{R L} D^{\beta} \underline{f}\right)\left(x_{0} ; r\right)\right] .
\end{aligned}
$$


If $\left({ }^{R L} D_{1}^{\beta} f\right)(x)$ is ${ }^{R L}[(i)-\beta]$-differentiable fuzzy-valued function, then for $1<\beta<2$

$$
\begin{aligned}
& \left({ }^{R L} D_{1,1}^{\beta} f\right)\left(x_{0}\right) \\
& \quad=\left[\left({ }^{R L} D^{\beta} \underline{f}\right)\left(x_{0} ; r\right),\left({ }^{R L} D^{\beta} \bar{f}\right)\left(x_{0} ; r\right)\right] .
\end{aligned}
$$

If $\left({ }^{R L} D_{1}^{\beta} f\right)(x)$ is ${ }^{R L}[(i i)-\beta]$-differentiable fuzzy-valued function, then for $1<\beta<2$

$$
\begin{aligned}
& \left({ }^{R L} D_{1,2}^{\beta} f\right)\left(x_{0}\right) \\
& \quad=\left[\left({ }^{R L} D^{\beta} \bar{f}\right)\left(x_{0} ; r\right),\left({ }^{R L} D^{\beta} \underline{f}\right)\left(x_{0} ; r\right)\right] .
\end{aligned}
$$

If $\left({ }^{R L} D_{2}^{\beta} f\right)(x)$ is ${ }^{R L}[(i)-\beta]$-differentiable fuzzy-valued function, then for $1<\beta<2$

$$
\begin{aligned}
& \left({ }^{R L} D_{2,1}^{\beta} f\right)\left(x_{0}\right) \\
& \quad=\left[\left({ }^{R L} D^{\beta} \bar{f}\right)\left(x_{0} ; r\right),\left({ }^{R L} D^{\beta} \underline{f}\right)\left(x_{0} ; r\right)\right] .
\end{aligned}
$$

If $\left({ }^{R L} D_{2}^{\beta} f\right)(x)$ is ${ }^{R L}[(i i)-\beta]$-differentiable fuzzy-valued function, then for $1<\beta<2$

$$
\begin{aligned}
& \left({ }^{R L} D_{2,2}^{\beta} f\right)\left(x_{0}\right) \\
& \quad=\left[\left({ }^{R L} D^{\beta} \underline{f}\right)\left(x_{0} ; r\right),\left({ }^{R L} D^{\beta} \bar{f}\right)\left(x_{0} ; r\right)\right] .
\end{aligned}
$$

If $\left({ }^{R L} D_{1,1}^{\beta} f\right)(x)$ is ${ }^{R L}[(i)-\beta]$-differentiable fuzzy-valued function, then for $2<\beta<3$

$$
\begin{aligned}
& \left({ }^{R L} D_{1,1,1}^{\beta} f\right)\left(x_{0}\right) \\
& \quad=\left[\left({ }^{R L} D^{\beta} \underline{f}\right)\left(x_{0} ; r\right),\left({ }^{R L} D^{\beta} \bar{f}\right)\left(x_{0} ; r\right)\right] .
\end{aligned}
$$

If $\left({ }^{R L} D_{1,1}^{\beta} f\right)(x)$ is ${ }^{R L}[(i i)-\beta]$-differentiable fuzzy-valued function, then for $2<\beta<3$

$$
\begin{aligned}
& \left({ }^{R L} D_{1,1,2}^{\beta} f\right)\left(x_{0}\right) \\
& \quad=\left[\left({ }^{R L} D^{\beta} \bar{f}\right)\left(x_{0} ; r\right),\left({ }^{R L} D^{\beta} \underline{f}\right)\left(x_{0} ; r\right)\right] .
\end{aligned}
$$

If $\left({ }^{R L} D_{1,2}^{\beta} f\right)(x)$ is ${ }^{R L}[(i)-\beta]$-differentiable fuzzy-valued function, then for $2<\beta<3$

$$
\begin{aligned}
& \left({ }^{R L} D_{1,2,1}^{\beta} f\right)\left(x_{0}\right) \\
& \quad=\left[\left({ }^{R L} D^{\beta} \bar{f}\right)\left(x_{0} ; r\right),\left({ }^{R L} D^{\beta} \underline{f}\right)\left(x_{0} ; r\right)\right] .
\end{aligned}
$$

If $\left({ }^{R L} D_{1,2}^{\beta} f\right)(x)$ is ${ }^{R L}[(i i)-\beta]$-differentiable fuzzy-valued function, then for $2<\beta<3$

$$
\begin{aligned}
& \left({ }^{R L} D_{1,2,2}^{\beta} f\right)\left(x_{0}\right) \\
& \quad=\left[\left({ }^{R L} D^{\beta} \underline{f}\right)\left(x_{0} ; r\right),\left({ }^{R L} D^{\beta} \bar{f}\right)\left(x_{0} ; r\right)\right] .
\end{aligned}
$$

If $\left({ }^{R L} D_{2,1}^{\beta} f\right)(x)$ is ${ }^{R L}[(i)-\beta]$-differentiable fuzzy-valued function, then for $2<\beta<3$

$$
\begin{aligned}
& \left({ }^{R L} D_{2,1,1}^{\beta} f\right)\left(x_{0}\right) \\
& \quad=\left[\left({ }^{R L} D^{\beta} \bar{f}\right)\left(x_{0} ; r\right),\left({ }^{R L} D^{\beta} \underline{f}\right)\left(x_{0} ; r\right)\right] .
\end{aligned}
$$

If $\left({ }^{R L} D_{2,1}^{\beta} f\right)(x)$ is ${ }^{R L}[(i i)-\beta]$-differentiable fuzzy-valued function, then for $2<\beta<3$

$$
\begin{aligned}
& \left({ }^{R L} D_{2,1,2}^{\beta} f\right)\left(x_{0}\right) \\
& \quad=\left[\left({ }^{R L} D^{\beta} \underline{f}\right)\left(x_{0} ; r\right),\left({ }^{R L} D^{\beta} \bar{f}\right)\left(x_{0} ; r\right)\right] .
\end{aligned}
$$

If $\left({ }^{R L} D_{2,2}^{\beta} f\right)(x)$ is ${ }^{R L}[(i)-\beta]$-differentiable fuzzy-valued function, then for $2<\beta<3$

$$
\begin{aligned}
& \left({ }^{R L} D_{2,2,1}^{\beta} f\right)\left(x_{0}\right) \\
& \quad=\left[\left({ }^{R L} D^{\beta} \underline{f}\right)\left(x_{0} ; r\right),\left({ }^{R L} D^{\beta} \bar{f}\right)\left(x_{0} ; r\right)\right] .
\end{aligned}
$$

If $\left({ }^{R L} D_{2,2}^{\beta} f\right)(x)$ is ${ }^{R L}[(i i)-\beta]$-differentiable fuzzy-valued function, then for $2<\beta<3$

$$
\begin{aligned}
& \left({ }^{R L} D_{2,2,2}^{\beta} f\right)\left(x_{0}\right) \\
& \quad=\left[\left({ }^{R L} D^{\beta} \bar{f}\right)\left(x_{0} ; r\right),\left({ }^{R L} D^{\beta} \underline{f}\right)\left(x_{0} ; r\right)\right],
\end{aligned}
$$

where

$$
\begin{aligned}
& \left({ }^{R L} D^{\beta} \underline{f}\right)\left(x_{0} ; r\right) \\
& \quad=\left[\frac{1}{\Gamma(\lceil\beta\rceil-\beta)}\left(\frac{d}{d x}\right)^{\lceil\beta\rceil} \int_{0}^{x} \frac{\underline{f}(t ; r) d t}{(x-t)^{1-\lceil\beta]+\beta}}\right]_{x=x_{0}}, \\
& \left({ }^{R L} D^{\beta \bar{f}}\right)\left(x_{0} ; r\right) \\
& \quad=\left[\frac{1}{\Gamma(\lceil\beta\rceil-\beta)}\left(\frac{d}{d x}\right)^{\lceil\beta\rceil} \int_{0}^{x} \frac{\bar{f}(t ; r) d t}{(x-t)^{1-\lceil\beta]+\beta}}\right]_{x=x_{0}} .
\end{aligned}
$$

Theorem 9. Suppose that $f(x) \in C^{F}[0, \infty) \cap L^{F}[0, \infty)$ is fuzzy-valued function; $f(x)=[f(x ; r), \bar{f}(x ; r)]$ for $r \in[0,1]$. One supposes that $n-1<\beta<n$ and $m$ is the number of repetitions of 2 among $i_{1}, i_{2}, \ldots, i_{n}$, say, $i_{k_{1}}, i_{k_{2}}, \ldots, i_{k_{m}}$, such that $k_{1}<k_{2}<\cdots<k_{m}$; that is, $i_{k_{1}}=i_{k_{2}}=\cdots=i_{k_{m}}=2$ and $0 \leq$ $m \leq n$. Then, one has the following:

If $m$ is an even number, we have:

$$
\begin{aligned}
& L\left[\left({ }^{R L} D_{i_{1}, i_{2}, \ldots, i_{n}}^{\beta} f\right)(x)\right] \\
& =s^{\beta} L[f(x)] \ominus s^{n-1}\left({ }^{R L} D^{\beta-n} f\right)(0) \\
& \quad \otimes \sum_{k=0}^{n-2} s^{k}\left({ }^{R L} D^{\beta-k-1} f\right)(0)
\end{aligned}
$$


such that

$$
\otimes= \begin{cases}\ominus, & \text { if the number of repetitions of } 2 \text { among } i_{1}, i_{2}, \ldots, i_{n-(k+1)} \text { is an even number }, \\ -, & \text { if the number of repetitions of } 2 \text { among } i_{1}, i_{2}, \ldots, i_{n-(k+1)} \text { is an odd number. }\end{cases}
$$

If $m$ is an odd number, we have:

$$
\otimes \sum_{k=0}^{n-2} s^{k}\left({ }^{R L} D^{\beta-k-1} f\right)(0)
$$

$$
\begin{aligned}
L & {\left[\left({ }^{R L} D_{i_{1}, i_{2}, \ldots, i_{n}}^{\beta} f\right)(x)\right] } \\
& =-s^{n-1}\left({ }^{R L} D^{\beta-n} f\right)(0) \ominus\left(-s^{\beta}\right) L[f(x)]
\end{aligned}
$$

such that

$$
\otimes= \begin{cases}\ominus, & \text { if the number of repetitions of } 2 \text { among } i_{1}, i_{2}, \ldots, i_{n-(k+1)} \text { is an odd number }, \\ -, & \text { if the number of repetitions of } 2 \text { among } i_{1}, i_{2}, \ldots, i_{n-(k+1)} \text { is an even number. }\end{cases}
$$

Proof. By

$$
\left({ }^{\mathrm{RL}} D_{i_{1}, i_{2}, \ldots, i_{n}}^{\beta} f\right)(x)
$$

we

mean

$\left({ }^{\mathrm{RL}} D_{i_{1}, \ldots, i_{k_{1}}, \ldots, i_{k_{2}}, \ldots, i_{k_{m}}, \ldots, i_{n}}^{\beta} f\right)(x)$. Suppose that $m$ is an odd number; then, from Theorem 7, when $n-1<\beta<n$, we get:

$$
\begin{aligned}
& \left({ }^{\mathrm{RL}} D_{i_{1}, i_{2}, \ldots, i_{n}}^{\beta} f\right)(x) \\
& \quad=\left[\left({ }^{\mathrm{RL}} D^{\beta} \bar{f}\right)(x ; r),\left({ }^{\mathrm{RL}} D^{\beta} \underline{f}\right)(x ; r)\right] .
\end{aligned}
$$

Therefore, we get:

$$
\begin{aligned}
& \left(\underline{{ }^{\mathrm{RL}} D^{\beta} f}\right)(x ; r)=\left({ }^{\mathrm{RL}} D^{\beta} \bar{f}\right)(x ; r), \\
& \left(\overline{{ }^{\mathrm{RL}} D^{\beta} f}\right)(x ; r)=\left({ }^{\mathrm{RL}} D^{\beta} \underline{f}\right)(x ; r) .
\end{aligned}
$$

Then, from (54), we get:

$$
\begin{aligned}
L & {\left[\left({ }^{\mathrm{RL}} D_{i_{1}, i_{2}, \ldots, i_{n}}^{\beta} f\right)(x)\right] } \\
& =L\left[\left({ }^{\mathrm{RL}} D^{\beta} f\right)(x ; r),\left(\overline{{ }^{\mathrm{RL}} D^{\beta} f}\right)(x ; r)\right] \\
& =\left[\ell\left[\left({ }^{\mathrm{RL}} D^{\beta} \bar{f}\right)(x ; r)\right], \ell\left[\left({ }^{\mathrm{RL}} D^{\beta} \underline{f}\right)(x ; r)\right]\right] .
\end{aligned}
$$

We know from Laplace transform of the RiemannLiouville fractional derivative of order $\beta>0$ that

$$
\begin{aligned}
\ell[ & \left.\left({ }^{\mathrm{RL}} D^{\beta} \underline{f}\right)(x ; r)\right] \\
= & s^{\beta} \ell[\underline{f}(x ; r)]-\sum_{k=0}^{n-1} s^{k}\left({ }^{\mathrm{RL}} D^{\beta-k-1} \underline{f}\right)(0 ; r) \\
= & s^{\beta} \ell[\underline{f}(x ; r)]-s^{n-1}\left({ }^{\mathrm{RL}} D^{\beta-n} \underline{f}\right)(0 ; r) \\
& \quad-\sum_{k=0}^{n-2} s^{k}\left({ }^{\mathrm{RL}} D^{\beta-k-1} \underline{f}\right)(0 ; r)
\end{aligned}
$$

$$
\begin{gathered}
=s^{\beta} \ell[\underline{f}(x ; r)]-s^{n-1}\left({ }^{\mathrm{RL}} D^{\beta-n} \underline{f}\right)(0 ; r) \\
-\sum_{k=0}^{n-2} s^{n-2-k}\left({ }^{\mathrm{RL}} D^{\beta-n+k+1} \underline{f}\right)(0 ; r) .
\end{gathered}
$$

The above equation can be written as:

$$
\begin{aligned}
\ell\left[\left({ }^{\mathrm{RL}} D^{\beta} f\right)(x ; r)\right] & \\
= & s^{\beta} \ell[\underline{f}(x ; r)]-s^{n-1}\left({ }^{\mathrm{RL}} D^{\beta-n} \underline{f}\right)(0 ; r) \\
& -\sum_{k=0}^{k_{1}-2} s^{n-2-k}\left({ }^{\mathrm{RL}} D^{\beta-n+k+1} \underline{f}\right)(0 ; r) \\
& -\sum_{k=k_{1}-1}^{k_{2}-2} s^{n-2-k}\left({ }^{\mathrm{RL}} D^{\beta-n+k+1} \underline{f}\right)(0 ; r)-\cdots \\
& -\sum_{k=k_{m-1}-1}^{k_{m}-2} s^{n-2-k}\left({ }^{\mathrm{RL}} D^{\beta-n+k+1} \underline{f}\right)(0 ; r) \\
& -\sum_{k=k_{m}-1}^{n-2} s^{n-2-k}\left({ }^{\mathrm{RL}} D^{\beta-n+k+1} \underline{f}\right)(0 ; r) .
\end{aligned}
$$

In a similar manner, we can get:

$$
\begin{aligned}
\ell\left[\left({ }^{\mathrm{RL}} D^{\beta} \bar{f}\right)(x ; r)\right] & =s^{\beta} \ell[\bar{f}(x ; r)]-s^{n-1}\left({ }^{\mathrm{RL}} D^{\beta-n} \bar{f}\right)(0 ; r) \\
& -\sum_{k=0}^{k_{1}-2} s^{n-2-k}\left({ }^{\mathrm{RL}} D^{\beta-n+k+1} \bar{f}\right)(0 ; r) \\
& \quad-\sum_{k=k_{1}-1}^{k_{2}-2} s^{n-2-k}\left({ }^{\mathrm{RL}} D^{\beta-n+k+1} \bar{f}\right)(0 ; r)-\cdots
\end{aligned}
$$




$$
\begin{aligned}
& -\sum_{k=k_{m-1}-1}^{k_{m}-2} s^{n-2-k}\left({ }^{\mathrm{RL}} D^{\beta-n+k+1} \bar{f}\right)(0 ; r) \\
& -\sum_{k=k_{m}-1}^{n-2} s^{n-2-k}\left({ }^{\mathrm{RL}} D^{\beta-n+k+1} \bar{f}\right)(0 ; r) .
\end{aligned}
$$

Since $i_{k_{1}}=i_{k_{2}}=\cdots=i_{k_{m}}=2$ and $m$ is an odd number, then we have the following equations:

$$
\begin{aligned}
& \left({ }^{\mathrm{RL}} D^{\beta-n+k+1} \underline{f}\right)(0 ; r)=\left(\underline{{ }^{\mathrm{RL}} D^{\beta-n+k+1} f}\right)(0 ; r) \text {, } \\
& \left({ }^{\mathrm{RL}} D^{\beta-n+k+1} \bar{f}\right)(0 ; r)=\left(\overline{{ }^{\mathrm{RL}} D^{\beta-n+k+1} f}\right)(0 ; r), \\
& \text { for } 0 \leq k \leq k_{1}-2 \text {, } \\
& \left({ }^{\mathrm{RL}} D^{\beta-n+k+1} \underline{f}\right)(0 ; r)=\left(\overline{{ }^{\mathrm{RL}} D^{\beta-n+k+1} f}\right)(0 ; r), \\
& \left({ }^{\mathrm{RL}} D^{\beta-n+k+1} \bar{f}\right)(0 ; r)=\left({ }^{\mathrm{RL}} D^{\beta-n+k+1} f\right)(0 ; r), \\
& \text { for } k_{1}-1 \leq k \leq k_{2}-2 \text {, } \\
& \vdots \\
& \left({ }^{\mathrm{RL}} D^{\beta-n+k+1} \underline{f}\right)(0 ; r)=\left(\underline{{ }^{\mathrm{RL}} D^{\beta-n+k+1} f}\right)(0 ; r), \\
& \left({ }^{\mathrm{RL}} D^{\beta-n+k+1} \bar{f}\right)(0 ; r)=\left(\overline{{ }^{\mathrm{RL}} D^{\beta-n+k+1} f}\right)(0 ; r), \\
& \text { for } k_{m-1}-1 \leq k \leq k_{m}-2 \text {, } \\
& \left({ }^{\mathrm{RL}} D^{\beta-n+k+1} \underline{f}\right)(0 ; r)=\left(\overline{{ }^{\mathrm{RL}} D^{\beta-n+k+1} f}\right)(0 ; r), \\
& \left({ }^{\mathrm{RL}} D^{\beta-n+k+1} \bar{f}\right)(0 ; r)=\left(\underline{{ }^{\mathrm{RL}} D^{\beta-n+k+1} f}\right)(0 ; r), \\
& \text { for } k_{m}-1 \leq k \leq n-2 \text {. }
\end{aligned}
$$

The last one of the above equations yields from Theorem 7 because $m$ is an odd number. Using (57), (58), and the above equations, (55) becomes:

$$
\begin{aligned}
L\left[\left({ }^{\mathrm{RL}} D_{i_{1}, i_{2}, \ldots, i_{n}}^{\beta} f\right)(x)\right] & -s^{n-1}\left({ }^{\mathrm{RL}} D^{\beta-n} f\right)(0) \ominus\left(-s^{\beta}\right) L[f(x)] \\
& \otimes \sum_{k=0}^{n-2} s^{n-2-k}\left({ }^{\mathrm{RL}} D^{\beta-n+k+1} f\right)(0) \\
= & -s^{n-1}\left({ }^{\mathrm{RL}} D^{\beta-n} f\right)(0) \ominus\left(-s^{\beta}\right) L[f(x)] \\
& \otimes \sum_{k=0}^{n-2} s^{k}\left({ }^{\mathrm{RL}} D^{\beta-k-1} f\right)(0),
\end{aligned}
$$

where $\otimes$ is defined as in (52).

If $m$ is an even number, the proof is similar.

We note that if we take $n=1(0<\beta<1)$ in Theorem 9, we get Theorem 4.4 [2] which is found by Salahshour et al.
Corollary 10. Suppose that $f(x) \in C^{F}[0, \infty) \cap L^{F}[0, \infty)$. One supposes that $2<\beta<3$. Then, one finds the following.

If $\left({ }^{R L} D_{1,1}^{\beta} f\right)(x)$ is ${ }^{R L}[(i)-\beta]$-differentiable fuzzy-valued function, then

$$
\begin{aligned}
L\left[\left({ }^{R L} D_{1,1,1}^{\beta} f\right)(x)\right]= & s^{\beta} L[f(x)] \ominus s^{2}\left({ }^{R L} D^{\beta-3} f\right)(0) \\
& \ominus\left({ }^{R L} D^{\beta-1} f\right)(0) \\
& \ominus s\left({ }^{R L} D^{\beta-2} f\right)(0) .
\end{aligned}
$$

If $\left({ }^{R L} D_{1,1}^{\beta} f\right)(x)$ is ${ }^{R L}[(i i)-\beta]$-differentiable fuzzy-valued function, then

$$
\begin{aligned}
L\left[\left({ }^{R L} D_{1,1,2}^{\beta}\right)(x)\right]= & -s^{2}\left({ }^{R L} D^{\beta-3} f\right)(0) \\
& \ominus\left(-s^{\beta}\right) L[f(x)] \\
& -\left({ }^{R L} D^{\beta-1} f\right)(0) \\
& -s\left({ }^{R L} D^{\beta-2} f\right)(0) .
\end{aligned}
$$

If $\left({ }^{R L} D_{1,2}^{\beta} f\right)(x)$ is ${ }^{R L}[(i)-\beta]$-differentiable fuzzy-valued function, then

$$
\begin{aligned}
L\left[\left({ }^{R L} D_{1,2,1}^{\beta} f\right)(x)\right]= & -s^{2}\left({ }^{R L} D^{\beta-3} f\right)(0) \\
& \ominus\left(-s^{\beta}\right) L[f(x)] \\
& \ominus\left({ }^{R L} D^{\beta-1} f\right)(0) \\
& -s\left({ }^{R L} D^{\beta-2} f\right)(0) .
\end{aligned}
$$

If $\left({ }^{R L} D_{1,2}^{\beta} f\right)(x)$ is ${ }^{R L}[(i i)-\beta]$-differentiable fuzzy-valued function, then

$$
\begin{aligned}
L\left[\left({ }^{R L} D_{1,2,2}^{\beta} f\right)(x)\right]= & s^{\beta} L[f(x)] \ominus s^{2}\left({ }^{R L} D^{\beta-3} f\right)(0) \\
& -\left({ }^{R L} D^{\beta-1} f\right)(0) \\
& \ominus s\left({ }^{R L} D^{\beta-2} f\right)(0) .
\end{aligned}
$$

If $\left({ }^{R L} D_{2,1}^{\beta} f\right)(x)$ is ${ }^{R L}[(i)-\beta]$-differentiable fuzzy-valued function, then

$$
\begin{aligned}
L\left[\left({ }^{R L} D_{2,1,1}^{\beta} f\right)(x)\right]= & -s^{2}\left({ }^{R L} D^{\beta-3} f\right)(0) \\
& \ominus\left(-s^{\beta}\right) L[f(x)] \\
& \ominus\left({ }^{R L} D^{\beta-1} f\right)(0) \\
& \ominus s\left({ }^{R L} D^{\beta-2} f\right)(0) .
\end{aligned}
$$


If $\left({ }^{R L} D_{2,1}^{\beta} f\right)(x)$ is ${ }^{R L}[(i i)-\beta]$-differentiable fuzzy-valued function, then

$$
\begin{aligned}
L\left[\left({ }^{R L} D_{2,1,2}^{\beta} f\right)(x)\right]= & s^{\beta} L[f(x)] \ominus s^{2}\left({ }^{R L} D^{\beta-3} f\right)(0) \\
& -\left({ }^{R L} D^{\beta-1} f\right)(0) \\
& -s\left({ }^{R L} D^{\beta-2} f\right)(0)
\end{aligned}
$$

If $\left({ }^{R L} D_{2,2}^{\beta} f\right)(x)$ is ${ }^{R L}[(i)-\beta]$-differentiable fuzzy-valued function, then

$$
\begin{aligned}
L\left[\left({ }^{R L} D_{2,2,1}^{\beta} f\right)(x)\right]= & s^{\beta} L[f(x)] \ominus s^{2}\left({ }^{R L} D^{\beta-3} f\right)(0) \\
& \ominus\left({ }^{R L} D^{\beta-1} f\right)(0) \\
& -s\left({ }^{R L} D^{\beta-2} f\right)(0)
\end{aligned}
$$

If $\left({ }^{R L} D_{2,2}^{\beta} f\right)(x)$ is ${ }^{R L}[(i i)-\beta]$-differentiable fuzzy-valued function, then

$$
\begin{aligned}
L\left[\left({ }^{R L} D_{2,2,2}^{\beta} f\right)(x)\right]= & -s^{2}\left({ }^{R L} D^{\beta-3} f\right)(0) \\
& \ominus\left(-s^{\beta}\right) L[f(x)] \\
& -\left({ }^{R L} D^{\beta-1} f\right)(0) \\
& \ominus s\left({ }^{R L} D^{\beta-2} f\right)(0) .
\end{aligned}
$$

Example 11. Consider the following FFIVP:

$$
\begin{gathered}
\left({ }^{\mathrm{RL}} D^{\beta} y\right)(x)=\sigma ; \\
\sigma=(2 r-2,2-2 r), 1<\beta<2, \\
\left({ }^{\mathrm{RL}} D^{\beta-1} y\right)(0)=\left({ }^{\mathrm{RL}} D^{\beta-2} y\right)(0)={ }^{\mathrm{RL}} y_{0}^{(\beta-1)} \in E .
\end{gathered}
$$

We note that

$$
\begin{aligned}
& \left(\underline{{ }^{\mathrm{RL}} D^{\beta-1} y}\right)(0 ; r)=\left({ }^{\mathrm{RL}} D^{\beta-2} \underline{y}\right)(0 ; r)={ }^{\mathrm{RL}} \underline{y}_{0}^{(\beta-1)}(r), \\
& \left(\overline{{ }^{\mathrm{RL}} D^{\beta-1} y}\right)(0 ; r)=\left({ }^{\mathrm{RL}} D^{\beta-2} \bar{y}\right)(0 ; r)={ }^{\mathrm{RL}} \bar{y}_{0}^{(\beta-1)}(r) .
\end{aligned}
$$

By taking fuzzy Laplace transform for both sides of (69), we get

$$
L\left[\left({ }^{\mathrm{RL}} D^{\beta} y\right)(x)\right]=L[\sigma]
$$

Now, by using Theorem 9 when $n=2$ we have $2^{2}=4$ cases as follows.

Case 1. Let $\left({ }^{\mathrm{RL}} D_{1}^{\beta} y\right)(x)$ be ${ }^{\mathrm{RL}}[i-\beta]$-differentiable. By using Theorem 9, when $m=0$ (even), (72) becomes

$$
\begin{aligned}
s^{\beta} L & {[y(x)] \ominus s\left({ }^{\mathrm{RL}} D^{\beta-2} y\right)(0) \ominus\left({ }^{\mathrm{RL}} D^{\beta-1} y\right)(0) } \\
= & L[\sigma] .
\end{aligned}
$$

Then, we get

$$
\begin{aligned}
& s^{\beta} \ell[\underline{y}(x ; r)]-s\left({ }^{\mathrm{RL}} D^{\beta-2} \underline{y}\right)(0 ; r)-\left(\underline{{ }^{\mathrm{RL}} D^{\beta-1} y}\right)(0 ; r) \\
& =\frac{2 r-2}{s}, \\
& s^{\beta} \ell[\bar{y}(x ; r)]-s\left({ }^{\mathrm{RL}} D^{\beta-2} \bar{y}\right)(0 ; r)-\left(\overline{{ }^{\mathrm{RL}} D^{\beta-1} y}\right)(0 ; r) \\
& =\frac{2-2 r}{s} .
\end{aligned}
$$

The solution of FFIVP (69) is as follows:

$$
\begin{aligned}
\underline{y}(x ; r)= & (2 r-2) \frac{x^{\beta}}{\Gamma(\beta+1)} \\
& +{ }^{\mathrm{RL}} \underline{y}_{0}^{(\beta-1)}(r)\left(\frac{x^{\beta-1}}{\Gamma(\beta)}+\frac{x^{\beta-2}}{\Gamma(\beta-1)}\right), \\
\bar{y}(x ; r)= & (2-2 r) \frac{x^{\beta}}{\Gamma(\beta+1)} \\
& +{ }^{\mathrm{RL}} \bar{y}_{0}^{(\beta-1)}(r)\left(\frac{x^{\beta-1}}{\Gamma(\beta)}+\frac{x^{\beta-2}}{\Gamma(\beta-1)}\right) .
\end{aligned}
$$

Case 2. Let $\left({ }^{\mathrm{RL}} D_{1}^{\beta} y\right)(x)$ be ${ }^{\mathrm{RL}}[i i-\beta]$-differentiable. By using Theorem 9, when $m=1$ (odd), (72) becomes

$$
\begin{aligned}
& -s\left({ }^{\mathrm{RL}} D^{\beta-2} y\right)(0) \ominus\left(-s^{\beta}\right) L[y(x)]-\left({ }^{\mathrm{RL}} D^{\beta-1} y\right)(0) \\
& =L[\sigma] .
\end{aligned}
$$

Then, we get:

$$
\begin{gathered}
-s\left({ }^{\mathrm{RL}} D^{\beta-2} \underline{y}\right)(0 ; r)+s^{\beta} \ell[y(x ; r)] \\
-\left({ }^{\mathrm{RL}} D^{\beta-1} y\right)(0 ; r)=\frac{2-2 r}{s}, \\
-s\left({ }^{\mathrm{RL}} D^{\beta-2} \bar{y}\right)(0 ; r)+s^{\beta} \ell[\bar{y}(x ; r)] \\
-\left(\overline{{ }^{\mathrm{RL}} D^{\beta-1} y}\right)(0 ; r)=\frac{2 r-2}{s} .
\end{gathered}
$$

The solution of FFIVP (69) is as follows:

$$
\begin{aligned}
\underline{y}(x ; r)= & (2-2 r) \frac{x^{\beta}}{\Gamma(\beta+1)} \\
& +{ }^{\mathrm{RL}} \underline{y}_{0}^{(\beta-1)}(r)\left(\frac{x^{\beta-1}}{\Gamma(\beta)}+\frac{x^{\beta-2}}{\Gamma(\beta-1)}\right), \\
\bar{y}(x ; r)= & (2 r-2) \frac{x^{\beta}}{\Gamma(\beta+1)} \\
& +{ }^{\mathrm{RL}} \bar{y}_{0}^{(\beta-1)}(r)\left(\frac{x^{\beta-1}}{\Gamma(\beta)}+\frac{x^{\beta-2}}{\Gamma(\beta-1)}\right) .
\end{aligned}
$$


Case 3. Let $\left({ }^{\mathrm{RL}} D_{2}^{\beta} y\right)(x)$ be ${ }^{\mathrm{RL}}[i-\beta]$-differentiable. By using Theorem 9, when $m=1$ (odd), (72) becomes:

$$
\begin{aligned}
- & s\left({ }^{\mathrm{RL}} D^{\beta-2} y\right)(0) \ominus\left(-s^{\beta}\right) L[y(x)] \ominus\left({ }^{\mathrm{RL}} D^{\beta-1} y\right)(0) \\
& =L[\sigma]
\end{aligned}
$$

Then, we get:

$$
\begin{gathered}
-s\left({ }^{\mathrm{RL}} D^{\beta-2} \underline{y}\right)(0 ; r)+s^{\beta} \ell[\underline{y}(x ; r)] \\
-\left(\overline{{ }^{\mathrm{RL}} D^{\beta-1} y}\right)(0 ; r)=\frac{2-2 r}{s}, \\
-s\left({ }^{\mathrm{RL}} D^{\beta-2} \bar{y}\right)(0 ; r)+s^{\beta} \ell[\bar{y}(x ; r)] \\
-\left(\frac{{ }^{\mathrm{RL}} D^{\beta-1} y}{}\right)(0 ; r)=\frac{2 r-2}{s} .
\end{gathered}
$$

The solution of FFIVP (69) is as follows:

$$
\begin{aligned}
\underline{y}(x ; r)= & (2-2 r) \frac{x^{\beta}}{\Gamma(\beta+1)}+{ }^{\mathrm{RL}} \bar{y}_{0}^{(\beta-1)}(r) \frac{x^{\beta-1}}{\Gamma(\beta)} \\
& +{ }^{\mathrm{RL}} \underline{y}_{0}^{(\beta-1)}(r) \frac{x^{\beta-2}}{\Gamma(\beta-1)}, \\
\bar{y}(x ; r)= & (2 r-2) \frac{x^{\beta}}{\Gamma(\beta+1)}+{ }^{\mathrm{RL}} \underline{y}_{0}^{(\beta-1)}(r) \frac{x^{\beta-1}}{\Gamma(\beta)} \\
& +{ }^{\mathrm{RL}} \bar{y}_{0}^{(\beta-1)}(r) \frac{x^{\beta-2}}{\Gamma(\beta-1)} .
\end{aligned}
$$

Case 4. Let $\left({ }^{\mathrm{RL}} D_{2}^{\beta} y\right)(x)$ be ${ }^{\mathrm{RL}}[i i-\beta]$-differentiable. By using Theorem 9, when $m=2$ (even), (72) becomes:

$$
\begin{aligned}
s^{\beta} L & {[y(x)] \ominus s\left({ }^{\mathrm{RL}} D^{\beta-2} y\right)(0)-\left({ }^{\mathrm{RL}} D^{\beta-1} y\right)(0) } \\
& =L[\sigma] .
\end{aligned}
$$

Then, we get:

$$
\begin{aligned}
& s^{\beta} \ell[\underline{y}(x ; r)]-s\left({ }^{\mathrm{RL}} D^{\beta-2} \underline{y}\right)(0 ; r)-\left(\overline{\mathrm{RL}^{\beta-1} y}\right)(0 ; r) \\
& =\frac{2 r-2}{s}, \\
& s^{\beta} \ell[\bar{y}(x ; r)]-s\left({ }^{\mathrm{RL}} D^{\beta-2} \bar{y}\right)(0 ; r)-\left(\underline{{ }^{\mathrm{RL}} D^{\beta-1} y}\right)(0 ; r) \\
& =\frac{2-2 r}{s} .
\end{aligned}
$$

The solution of FFIVP (69) is as follows:

$$
\begin{aligned}
\underline{y}(x ; r)= & (2 r-2) \frac{x^{\beta}}{\Gamma(\beta+1)}+{ }^{\mathrm{RL}} \bar{y}_{0}^{(\beta-1)}(r) \frac{x^{\beta-1}}{\Gamma(\beta)} \\
& +{ }^{\mathrm{RL}} \underline{y}_{0}^{(\beta-1)}(r) \frac{x^{\beta-2}}{\Gamma(\beta-1)},
\end{aligned}
$$

$$
\begin{aligned}
\bar{y}(x ; r)= & (2-2 r) \frac{x^{\beta}}{\Gamma(\beta+1)}+{ }^{\mathrm{RL}} \underline{y}_{0}^{(\beta-1)}(r) \frac{x^{\beta-1}}{\Gamma(\beta)} \\
& +{ }^{\mathrm{RL}} \bar{y}_{0}^{(\beta-1)}(r) \frac{x^{\beta-2}}{\Gamma(\beta-1)} .
\end{aligned}
$$

\section{Generalization of Fuzzy Laplace Transforms of the Fuzzy Caputo Fractional Derivatives of Order $n-1<\beta<n$}

In this section, we define Caputo fractional derivatives of the general fractional order $0<\beta<n$ and we find fuzzy Laplace transforms for Caputo fractional derivatives of the general fractional order $n-1<\beta<n$ for fuzzy-valued function $f$ under H-differentiability.

Remark 12. To get Caputo type fuzzy fractional derivatives of order $0<\beta<n$ for $f(x) \in C^{F}[0, b] \cap L^{F}[0, b]$, we take

$$
\begin{aligned}
G(x)= & \frac{1}{\Gamma([\beta\rceil-\beta)} \int_{0}^{x} \frac{f(t) d t}{(x-t)^{1-\lceil\beta\rceil+\beta}} \\
& \ominus \sum_{k=0}^{\lfloor\beta\rfloor} \frac{D^{k} f(0) x^{\lceil\beta\rceil-\beta+k}}{\Gamma(1+\lceil\beta\rceil-\beta+k)}
\end{aligned}
$$

instead of $\phi(x)=(1 / \Gamma(\lceil\beta\rceil-\beta)) \int_{0}^{x}\left(f(t) d t /(x-t)^{1-\lceil\beta\rceil+\beta}\right)$ in Definition 6.

We note that if we take $n=2(0<\beta<2)$ in Remark 12, we get Definition 3.1 [1] which is introduced by Mazandarani and Kamyad.

Theorem 13. Let $f(x) \in C^{F}[0, b] \cap L^{F}[0, b]$ be a fuzzy-valued function such that $f(x)=[f(x ; r), \bar{f}(x ; r)]$ for $r \in[0,1], x_{0} \in$ $(0, b)$, and $G(x)$ is defined as in (85).

Suppose that $0<\beta<n$ and $m$ is the number of repetitions of number 2 among $i_{1}, i_{2}, \ldots, i_{\lceil\beta\rceil}$ for $k-1<\beta<k, k=$ $1,2, \ldots, n$, say, $i_{k_{1}}, i_{k_{2}}, \ldots, i_{k_{m}}$, such that $k_{1}<k_{2}<\cdots<k_{m}$; that is, $i_{k_{1}}=i_{k_{2}}=\cdots=i_{k_{m}}=2$ and $0 \leq m \leq\lceil\beta\rceil$. Then, one can find the following:

If $m$ is an even number, then

$$
\begin{aligned}
& \left({ }^{C} D_{i_{1}, i_{2}, \ldots, i_{\Gamma \beta]}}^{\beta} f\right)\left(x_{0}\right) \\
& \quad=\left[\left({ }^{C} D^{\beta} \underline{f}\right)\left(x_{0} ; r\right),\left({ }^{C} D^{\beta} \bar{f}\right)\left(x_{0} ; r\right)\right] .
\end{aligned}
$$


If $m$ is an odd number, then

$$
\begin{aligned}
& \left({ }^{C} D_{i_{1}, i_{2}, \ldots, i_{[\beta]}}^{\beta} f\right)\left(x_{0}\right) \\
& \quad=\left[\left({ }^{C} D^{\beta} \bar{f}\right)\left(x_{0} ; r\right),\left({ }^{C} D^{\beta} \underline{f}\right)\left(x_{0} ; r\right)\right],
\end{aligned}
$$

where

$$
\begin{aligned}
& \left({ }^{C} D^{\beta} \underline{f}\right)\left(x_{0} ; r\right) \\
& =\left[\frac{1}{\Gamma(\lceil\beta\rceil-\beta)} \int_{0}^{x} \frac{D^{\lceil\beta\rceil} f(t ; r)}{(x-t)^{1-\lceil\beta\rceil+\beta}} d t\right]_{x=x_{0}}, \\
& \left({ }^{C} D^{\beta} \bar{f}\right)\left(x_{0} ; r\right) \\
& =\left[\frac{1}{\Gamma(\lceil\beta\rceil-\beta)} \int_{0}^{x} \frac{D^{\lceil\beta\rceil} \bar{f}(t ; r)}{(x-t)^{1-\lceil\beta\rceil+\beta}} d t\right]_{x=x_{0}}, \\
& D^{k} f(t)=\frac{d^{k} f(t)}{d t^{k}} .
\end{aligned}
$$

Proof. Let $m$ be an even number. If we make the same steps in the proof of Theorem 7 when $m$ is an even number, we get

$$
\left({ }^{C} D^{\beta} f\right)\left(x_{0}\right)=\left[D^{\lceil\beta\rceil} \underline{G}\left(x_{0} ; r\right), D^{\lceil\beta\rceil} \bar{G}\left(x_{0} ; r\right)\right],
$$

where $D=d / d x$.

Thus,

$$
\begin{aligned}
\left({ }^{C} D^{\beta} f\right)\left(x_{0}\right) & \\
= & {\left[D ^ { \lceil \beta \rceil } \left(\frac{1}{\Gamma(\lceil\beta\rceil-\beta)} \int_{0}^{x} \frac{\underline{f}(t ; r) d t}{(x-t)^{1-\lceil\beta\rceil+\beta}}\right.\right.} \\
& \left.-\sum_{k=0}^{\lfloor\beta\rfloor} \frac{D^{k} \underline{f}(0 ; r) x^{\lceil\beta\rceil-\beta+k}}{\Gamma(1+\lceil\beta\rceil-\beta+k)}\right)\left.\right|_{x=x_{0}}, \\
& D^{\lceil\beta\rceil}\left(\frac{1}{\Gamma(\lceil\beta\rceil-\beta)} \int_{0}^{x} \frac{\bar{f}(t ; r) d t}{(x-t)^{1-\lceil\beta\rceil+\beta}}\right. \\
& \left.\left.-\sum_{k=0}^{\lfloor\beta\rfloor} \frac{D^{k} \bar{f}(0 ; r) x^{\lceil\beta\rceil-\beta+k}}{\Gamma(1+\lceil\beta\rceil-\beta+k)}\right)\left.\right|_{x=x_{0}}\right] .
\end{aligned}
$$

By using Definition 5, we have:

$$
\begin{gathered}
\left({ }^{C} D^{\beta} f\right)\left(x_{0}\right)=\left[D^{\lceil\beta\rceil}\left(D^{-(\lceil\beta\rceil-\beta)} \underline{f}\right)\left(x_{0} ; r\right)\right. \\
-\left.\left(\sum_{k=0}^{\lfloor\beta\rfloor} \frac{D^{k} \underline{f}(0 ; r) D^{\lceil\beta\rceil} x^{\lceil\beta\rceil-\beta+k}}{\Gamma(1+\lceil\beta\rceil-\beta+k)}\right)\right|_{x=x_{0}},
\end{gathered}
$$

$$
\begin{aligned}
& D^{\lceil\beta\rceil}\left(D^{-(\lceil\beta\rceil-\beta)} \bar{f}\right)\left(x_{0} ; r\right) \\
& \left.-\left.\left(\sum_{k=0}^{\lfloor\beta\rfloor} \frac{D^{k} \bar{f}(0 ; r) D^{\lceil\beta\rceil} x^{\lceil\beta\rceil-\beta+k}}{\Gamma(1+\lceil\beta\rceil-\beta+k)}\right)\right|_{x=x_{0}}\right],
\end{aligned}
$$

where $\left(D^{-([\beta\rceil-\beta)} f\right)\left(x_{0} ; r\right)$ and $\left(D^{-(\lceil\beta\rceil-\beta)} \bar{f}\right)\left(x_{0} ; r\right)$ are the Riemann-Liouville fractional integrals of the functions $f(x ; r)$ and $\bar{f}(x ; r)$ at $x=x_{0}$, respectively. By using (b) of Theorem 1 with $n=\lceil\beta\rceil, \nu=\lceil\beta\rceil-\beta$, and the equation $D^{n} x^{m}=(\Gamma(m+1) / \Gamma(m+1-n)) x^{m-n}$, we get:

$$
\begin{aligned}
& \left({ }^{C} D^{\beta} f\right)\left(x_{0}\right)=\left[D^{-(\lceil\beta\rceil-\beta)}\left(D^{\lceil\beta\rceil} \underline{f}\left(x_{0} ; r\right)\right)\right. \\
& +\underline{Q}_{\lceil\beta\rceil}\left(x_{0},-\beta\right)-\left.\sum_{k=0}^{\lfloor\beta\rfloor} \frac{D^{k} \underline{f}(0 ; r) x^{k-\beta}}{\Gamma(1-\beta+k)}\right|_{x=x_{0}}, \\
& D^{-(\lceil\beta\rceil-\beta)}\left(D^{\lceil\beta\rceil} \bar{f}\left(x_{0} ; r\right)\right)+\bar{Q}_{\lceil\beta\rceil}\left(x_{0},-\beta\right) \\
& \left.-\left.\sum_{k=0}^{\lfloor\beta\rfloor} \frac{D^{k} \bar{f}(0 ; r) x^{k-\beta}}{\Gamma(1-\beta+k)}\right|_{x=x_{0}}\right] .
\end{aligned}
$$

Thus,

$$
\begin{aligned}
& \left({ }^{C} D^{\beta} f\right)\left(x_{0}\right)=\left[D^{-(\lceil\beta\rceil-\beta)}\left(D^{\lceil\beta\rceil} \underline{f}\left(x_{0} ; r\right)\right),\right. \\
& \left.D^{-(\lceil\beta\rceil-\beta)}\left(D^{\lceil\beta\rceil} \bar{f}\left(x_{0} ; r\right)\right)\right]=\left[\left({ }^{C} D^{\beta} \underline{f}\right)\left(x_{0} ; r\right),\right. \\
& \left.\left({ }^{C} D^{\beta} \bar{f}\right)\left(x_{0} ; r\right)\right] .
\end{aligned}
$$

If $m$ is an odd number, the proof is similar.

We note that if we take $n=2(0<\beta<2)$ in Theorem 13 we get Theorem 3.1 [1] which is found by Mazandarani and Kamyad.

Theorem 14. Suppose that $f(x) \in C^{F}[0, \infty) \cap L^{F}[0, \infty)$ is fuzzy-valued function $f(x)=[f(x ; r), \bar{f}(x ; r)]$ for $r \in[0,1]$. One supposes that $n-1<\beta \overline{<} n$ and $m$ is the number of repetitions of 2 among $i_{1}, i_{2}, \ldots, i_{n}$, say, $i_{k_{1}}, i_{k_{2}}, \ldots, i_{k_{m}}$, such that $k_{1}<k_{2}<\cdots<k_{m}$; that is, $i_{k_{1}}=i_{k_{2}}=\cdots=i_{k_{m}}=2$ and $0 \leq m \leq n$. Then, one has the following.

If $m$ is an even number, we have:

$$
\begin{array}{r}
L\left[\left({ }^{C} D_{i_{1}, i_{2}, \ldots, i_{n}}^{\beta} f\right)(x)\right]= \\
s^{\beta} L[f(x)] \ominus s^{\beta-1} f(0) \\
\otimes \sum_{k=1}^{n-1} s^{\beta-(k+1)} f^{(k)}(0),
\end{array}
$$


such that

$$
\otimes=\left\{\begin{array}{l}
\ominus, \quad \text { if the number of repetitions of number } 2 \text { among } i_{1}, i_{2}, \ldots, i_{k} \text { is an even number }, \\
-, \quad \text { if the number of repetitions of number } 2 \text { among } i_{1}, i_{2}, \ldots, i_{k} \text { is an odd number. }
\end{array}\right.
$$

If $m$ is an odd number, we have:

$$
\otimes \sum_{k=1}^{n-1} s^{\beta-(k+1)} f^{(k)}(0)
$$

$$
L\left[\left({ }^{C} D_{i_{1}, i_{2}, \ldots, i_{n}}^{\beta} f\right)(x)\right]=-s^{\beta-1} f(0) \ominus\left(-s^{\beta}\right) L[f(x)]
$$

such that

$$
\otimes=\left\{\begin{array}{l}
\ominus, \quad \text { if the number of repetitions of number } 2 \text { among } i_{1}, i_{2}, \ldots, i_{k} \text { is an odd number, } \\
-, \quad \text { if the number of repetitions of number } 2 \text { among } i_{1}, i_{2}, \ldots, i_{k} \text { is an even number. }
\end{array}\right.
$$

Proof. By $\quad\left({ }^{C} D_{i_{1}, i_{2}, \ldots, i_{n}}^{\beta} f\right)(x) \quad$ we mean $\left({ }^{C} D_{i_{1}, \ldots, i_{k_{1}}, \ldots, i_{k_{2}}, \ldots, i_{k_{m}}, \ldots, i_{n}}^{\beta} f\right)(x)$. Suppose that $m$ is an odd number, and then from Theorem 13 , when $n-1<\beta<n$, we get:

$$
\left({ }^{C} D_{i_{1}, i_{2}, \ldots, i_{n}}^{\beta} f\right)(x)=\left[\left({ }^{C} D^{\beta} \bar{f}\right)(x ; r),\left({ }^{C} D^{\beta} \underline{f}\right)(x ; r)\right] .
$$

Therefore, we get:

$$
\begin{aligned}
& \left({ }^{C} D^{\beta} f\right)(x ; r)=\left({ }^{C} D^{\beta} \bar{f}\right)(x ; r), \\
& \left(\overline{{ }^{C} D^{\beta} f}\right)(x ; r)=\left({ }^{C} D^{\beta} \underline{f}\right)(x ; r) .
\end{aligned}
$$

Then, from (99), we get:

$$
\begin{aligned}
L & {\left[\left({ }^{C} D_{i_{1}, i_{2}, \ldots, i_{n}}^{\beta} f\right)(x)\right] } \\
& =L\left[\left({ }^{C} D^{\beta} f\right)(x ; r),\left(\overline{{ }^{C} D^{\beta} f}\right)(x ; r)\right] \\
& =\left[\ell\left[\left({ }^{C} D^{\beta} \bar{f}\right)(x ; r)\right], \ell\left[\left({ }^{C} D^{\beta} \underline{f}\right)(x ; r)\right]\right] .
\end{aligned}
$$

We know from Laplace transform of the Caputo fractional derivative of order $\beta>0$ that

$$
\begin{aligned}
\ell\left[\left({ }^{C} D^{\beta} \underline{f}\right)(x ; r)\right]= & s^{\beta} \ell[\underline{f}(x ; r)] \\
& -\sum_{k=0}^{n-1} s^{\beta-k-1} \underline{f}^{(k)}(0 ; r) \\
= & s^{\beta} \ell[\underline{f}(x ; r)]-s^{\beta-1} \underline{f}(0 ; r) \\
& -\sum_{k=1}^{n-1} s^{\beta-k-1} \underline{f}^{(k)}(0 ; r) .
\end{aligned}
$$

The above equation can be written as:

$$
\begin{aligned}
\ell\left[\left({ }^{C} D^{\beta} \underline{f}\right)(x ; r)\right]= & s^{\beta} \ell[\underline{f}(x ; r)]-s^{\beta-1} \underline{f}(0 ; r) \\
& -\sum_{k=1}^{k_{1}-1} s^{\beta-k-1} \underline{f}^{(k)}(0 ; r) \\
& -\sum_{k=k_{1}}^{k_{2}-1} s^{\beta-k-1} \underline{f}^{(k)}(0 ; r)-\cdots \\
& -\sum_{k=k_{m-1}}^{k_{m}-1} s^{\beta-k-1} \underline{f}^{(k)}(0 ; r) \\
& -\sum_{k=k_{m}}^{n-1} s^{\beta-k-1} \underline{f}^{(k)}(0 ; r) .
\end{aligned}
$$

In a similar manner, we can get:

$$
\begin{aligned}
\ell\left[\left({ }^{C} D^{\beta} \bar{f}\right)(x ; r)\right]= & s^{\beta} \ell[\bar{f}(x ; r)]-s^{\beta-1} \bar{f}(0 ; r) \\
& -\sum_{k=1}^{k_{1}-1} s^{\beta-k-1} \bar{f}^{(k)}(0 ; r) \\
& -\sum_{k=k_{1}}^{k_{2}-1} s^{\beta-k-1} \bar{f}^{(k)}(0 ; r)-\cdots \\
& -\sum_{k=k_{m-1}}^{k_{m}-1} s^{\beta-k-1} \underline{f}^{(k)}(0 ; r) \\
& -\sum_{k=k_{m}}^{n-1} s^{\beta-k-1} \underline{f}^{(k)}(0 ; r) .
\end{aligned}
$$


Since $i_{k_{1}}=i_{k_{2}}=\cdots=i_{k_{m}}=2$ and $m$ is an odd number, we have the following equations:

$$
\begin{aligned}
& \underline{f}^{(k)}(0 ; r)=\underline{f^{(k)}}(0 ; r), \\
& \bar{f}^{(k)}(0 ; r)=\overline{f^{(k)}}(0 ; r) \text {; } \\
& 1 \leq k \leq k_{1}-1, \\
& \underline{f}^{(k)}(0 ; r)=\overline{f^{(k)}}(0 ; r), \\
& \bar{f}^{(k)}(0 ; r)=\underline{f}^{(k)}(0 ; r) ; \\
& k_{1} \leq k \leq k_{2}-1 \text {, } \\
& \text { : } \\
& \underline{f}^{(k)}(0 ; r)=\underline{f^{(k)}}(0 ; r), \\
& \bar{f}^{(k)}(0 ; r)=\overline{f^{(k)}}(0 ; r) \text {; } \\
& k_{m-1} \leq k \leq k_{m}-1, \\
& \underline{f}^{(k)}(0 ; r)=\overline{f^{(k)}}(0 ; r) \text {, } \\
& \bar{f}^{(k)}(0 ; r)=f^{(k)}(0 ; r) ; \\
& k_{m} \leq k \leq n-1 .
\end{aligned}
$$

The last one of the equations in (104) yields because $m$ is an odd number. Using (102), (103), and (104); then (100) becomes:

$$
\begin{aligned}
& L\left[\left({ }^{C} D_{i_{1}, i_{2}, \ldots, i_{n}}^{\beta} f\right)(x)\right] \\
& =-s^{\beta-1} f(0) \ominus\left(-s^{\beta}\right) L[f(x)] \\
& \quad \otimes \sum_{k=1}^{n-1} s^{\beta-k-1} f^{(k)}(0),
\end{aligned}
$$

where $\otimes$ is defined as (97).

If $m$ is an even number, the proof is similar.

Corollary 15. Suppose that $f(x) \in C^{F}[0, \infty) \cap L^{F}[0, \infty)$. One supposes that $2<\beta<3$. Then:

If $\left({ }^{C} D_{1,1}^{\beta} f\right)(x)$ is ${ }^{C}[i-\beta]$-differentiable fuzzy-valued function, then

$$
\begin{aligned}
L\left[\left({ }^{C} D_{1,1,1}^{\beta} f\right)(x)\right]= & s^{\beta} L[f(x)] \ominus s^{\beta-1} f(0) \\
& \ominus s^{\beta-2} f^{\prime}(0) \ominus s^{\beta-3} f^{\prime \prime}(0) .
\end{aligned}
$$

If $\left({ }^{C} D_{1,1}^{\beta} f\right)(x)$ is ${ }^{C}[i i-\beta]$-differentiable fuzzy-valued function, then

$$
\begin{aligned}
L\left[\left({ }^{C} D_{1,1,2}^{\beta} f\right)(x)\right]= & -s^{\beta-1} f(0) \ominus\left(-s^{\beta}\right) L[f(x)] \\
& -s^{\beta-2} f^{\prime}(0)-s^{\beta-3} f^{\prime \prime}(0) .
\end{aligned}
$$

If $\left({ }^{C} D_{1,2}^{\beta} f\right)(x)$ is ${ }^{C}[i-\beta]$-differentiable fuzzy-valued function, then

$$
\begin{aligned}
L\left[\left({ }^{C} D_{1,2,1}^{\beta} f\right)(x)\right]= & -s^{\beta-1} f(0) \ominus\left(-s^{\beta}\right) L[f(x)] \\
& -s^{\beta-2} f^{\prime}(0) \ominus s^{\beta-3} f^{\prime \prime}(0) .
\end{aligned}
$$

If $\left({ }^{C} D_{1,2}^{\beta} f\right)(x)$ is ${ }^{C}[i i-\beta]$-differentiable fuzzy-valued function, then

$$
\begin{aligned}
L\left[\left({ }^{C} D_{1,2,2}^{\beta} f\right)(x)\right]= & s^{\beta} L[f(x)] \ominus s^{\beta-1} f(0) \\
& \ominus s^{\beta-2} f^{\prime}(0)-s^{\beta-3} f^{\prime \prime}(0) .
\end{aligned}
$$

If $\left({ }^{C} D_{2,1}^{\beta} f\right)(x)$ is ${ }^{C}[i-\beta]$-differentiable fuzzy-valued function, then

$$
\begin{aligned}
L\left[\left({ }^{C} D_{2,1,1}^{\beta} f\right)(x)\right]= & -s^{\beta-1} f(0) \ominus\left(-s^{\beta}\right) L[f(x)] \\
& \ominus s^{\beta-2} f^{\prime}(0) \ominus s^{\beta-3} f^{\prime \prime}(0) .
\end{aligned}
$$

If $\left({ }^{C} D_{2,1}^{\beta} f\right)(x)$ is ${ }^{C}[i i-\beta]$-differentiable fuzzy-valued function, then

$$
\begin{aligned}
L\left[\left({ }^{C} D_{2,1,2}^{\beta} f\right)(x)\right]= & s^{\beta} L[f(x)] \ominus s^{\beta-1} f(0) \\
& -s^{\beta-2} f^{\prime}(0)-s^{\beta-3} f^{\prime \prime}(0) .
\end{aligned}
$$

If $\left({ }^{C} D_{2,2}^{\beta} f\right)(x)$ is ${ }^{C}[i-\beta]$-differentiable fuzzy-valued function, then

$$
\begin{aligned}
L\left[\left({ }^{C} D_{2,2,1}^{\beta} f\right)(x)\right]= & s^{\beta} L[f(x)] \ominus s^{\beta-1} f(0) \\
& -s^{\beta-2} f^{\prime}(0) \ominus s^{\beta-3} f^{\prime \prime}(0) .
\end{aligned}
$$

If $\left({ }^{C} D_{2,2}^{\beta} f\right)(x)$ is ${ }^{C}[i i-\beta]$-differentiable fuzzy-valued function, then

$$
\begin{aligned}
L\left[\left({ }^{C} D_{2,2,2}^{\beta} f\right)(x)\right]= & -s^{\beta-1} f(0) \ominus\left(-s^{\beta}\right) L[f(x)] \\
& \ominus s^{\beta-2} f^{\prime}(0)-s^{\beta-3} f^{\prime \prime}(0) .
\end{aligned}
$$

Example 16. Consider the following FFIVP:

$$
\begin{aligned}
\left({ }^{C} D^{3 / 4} y\right)(x) & =-y(x), \quad 0<\beta<1, \\
y(0) & =(r, 2-r) .
\end{aligned}
$$

We note that:

$$
\begin{aligned}
& \underline{y}(0 ; r)=r, \\
& \bar{y}(0 ; r)=2-r .
\end{aligned}
$$

By taking fuzzy Laplace transform for both sides of (114), we get:

$$
L\left[\left({ }^{C} D^{3 / 4} y\right)(x)\right]=-L[y(x)] .
$$

Now, by using Theorem 14 when $n=1$ we have $2^{1}=2$ cases as follows. 
Case 1. Let $y(x)$ be ${ }^{C}[(i)-\beta]$-differentiable fuzzy-valued function. By using Theorem 14, when $m=0$ (even), (117) becomes:

$$
s^{3 / 4} L[y(x)] \ominus s^{-1 / 4} y(0)=-L[y(x)] .
$$

Then, we get:

$$
\begin{aligned}
& s^{3 / 4} \ell[\underline{y}(x ; r)]-s^{-1 / 4} \underline{y}(0 ; r)=-\ell[\bar{y}(x ; r)], \\
& s^{3 / 4} \ell[\bar{y}(x ; r)]-s^{-1 / 4} \bar{y}(0 ; r)=-\ell[\underline{y}(x ; r)] .
\end{aligned}
$$

The solution of the above system is

$$
\begin{aligned}
& \ell[\underline{y}(x ; r)]=\frac{r s^{1 / 2}-(2-r) s^{-1 / 4}}{s^{3 / 2}-1} \\
& \ell[\bar{y}(x ; r)]=\frac{(2-r) s^{1 / 2}-r s^{-1 / 4}}{s^{3 / 2}-1} .
\end{aligned}
$$

The solution of FFIVP (114) is as follows:

$$
\begin{aligned}
& \underline{y}(x ; r)=r E_{3 / 2,1}\left(x^{3 / 2}\right)-(2-r) x^{3 / 4} E_{3 / 2,7 / 4}\left(x^{3 / 2}\right), \\
& \bar{y}(x ; r)=(2-r) E_{3 / 2,1}\left(x^{3 / 2}\right)-r x^{3 / 4} E_{3 / 2,7 / 4}\left(x^{3 / 2}\right),
\end{aligned}
$$

where $E_{\alpha, \beta}(z)$ denotes the Mittag-Leffler function.

Case 2. Let $y(x)$ be ${ }^{C}[(i i)-\beta]$-differentiable fuzzy-valued function. By using Theorem 14, when $m=1$ (odd), (117) becomes:

$$
-s^{-1 / 4} y(0) \ominus\left(-s^{3 / 4}\right) L[y(x)]=-L[y(x)] .
$$

Then, we get:

$$
\begin{aligned}
& -s^{-1 / 4} \bar{y}(0 ; r)+s^{3 / 4} \ell[\bar{y}(x ; r)]=-\ell[\bar{y}(x ; r)], \\
& -s^{-1 / 4} \underline{y}(0 ; r)+s^{3 / 4} \ell[\underline{y}(x ; r)]=-\ell[\underline{y}(x ; r)] .
\end{aligned}
$$

The solution of FFIVP (114) is as follows:

$$
\begin{aligned}
& \underline{y}(x ; r)=r E_{3 / 4,1}\left(-x^{3 / 4}\right), \\
& \bar{y}(x ; r)=(2-r) E_{3 / 4,1}\left(-x^{3 / 4}\right) .
\end{aligned}
$$

\section{Conclusions}

The general formulas for fuzzy Riemann-Liouville and Caputo fractional derivatives about the general order $0<$ $\beta<n$ for fuzzy-valued function $f$ are found by using all the possible arrangements of objects such that $r_{1}$ of them equal 1 and $r_{2}$ (the others) of them equal 2. Also, the general formulas for fuzzy Laplace transforms of Riemann-Liouville and Caputo fractional derivatives about the general order $n-1<\beta<n$ are found under Hukuhara difference (H-difference).

\section{Conflict of Interests}

The authors declare that there is no conflict of interests regarding the publication of this paper.

\section{References}

[1] M. Mazandarani and A. V. Kamyad, "Modified fractional Euler method for solving fuzzy fractional initial value problem," Communications in Nonlinear Science and Numerical Simulation, vol. 18, no. 1, pp. 12-21, 2013.

[2] S. Salahshour, T. Allahviranloo, and S. Abbasbandy, "Solving fuzzy fractional differential equations by fuzzy Laplace transforms," Communications in Nonlinear Science and Numerical Simulation, vol. 17, no. 3, pp. 1372-1381, 2012.

[3] G.-C. Wu and D. Baleanu, "Variational iteration method for fractional calculus-a universal approach by Laplace transform," Advances in Difference Equations, vol. 2013, article 18, 2013.

[4] A. Ahmadian, M. Suleiman, S. Salahshour, and D. Baleanu, "A Jacobi operational matrix for solving a fuzzy linear fractional differential equation," Advances in Difference Equations, vol. 2013, article 104, 2013.

[5] T. Allahviranloo, A. Armand, and Z. Gouyandeh, "Fuzzy fractional differential equations under generalized fuzzy Caputo derivative," Journal of Intelligent and Fuzzy Systems, vol. 26, no. 3, pp. 1481-1490, 2014.

[6] K. S. Miller and B. Ross, Introduction to the Fractional Calculus and Fractional Differential Equations, John Wiley \& Sons, New York, NY, USA, 1993.

[7] T. Allahviranloo, S. Salahshour, and S. Abbasbandy, "Explicit solutions of fractional differential equations with uncertainty," Soft Computing, vol. 16, no. 2, pp. 297-302, 2012.

[8] S. Salahshour and T. Allahviranloo, "Applications of fuzzy laplace transforms," Soft Computing, vol. 17, no. 1, pp. 145-158, 2013.

[9] T. Allahviranloo and M. B. Ahmadi, "Fuzzy Laplace transforms," Soft Computing, vol. 14, no. 3, pp. 235-243, 2010. 


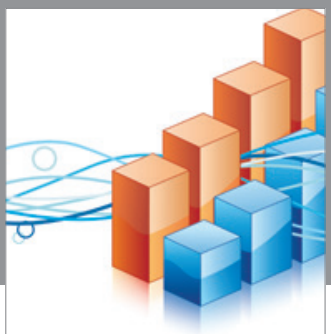

Advances in

Operations Research

vatem alat4

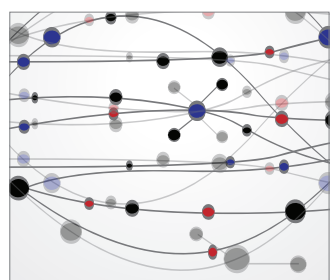

\section{The Scientific} World Journal
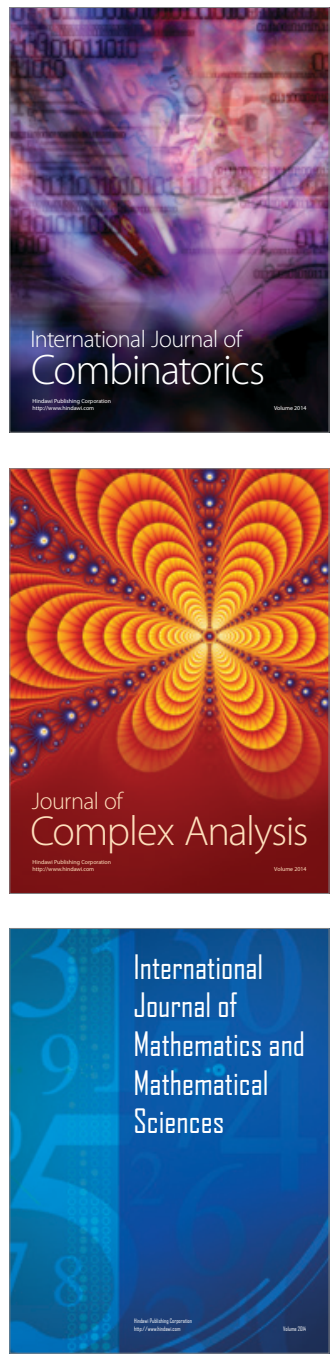
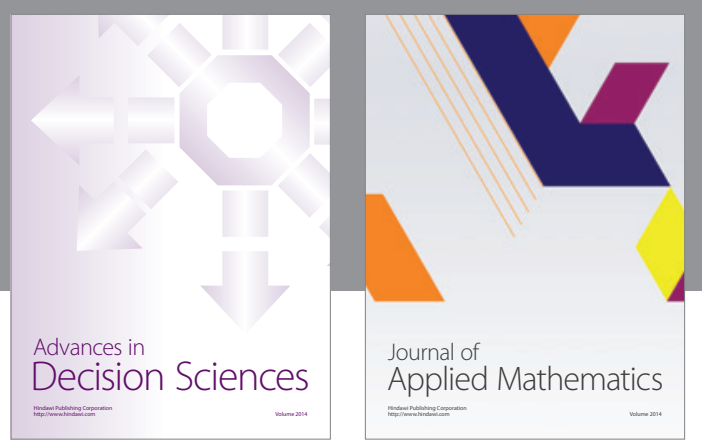

Algebra

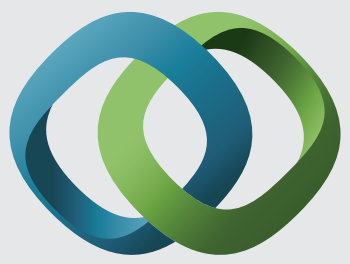

\section{Hindawi}

Submit your manuscripts at

http://www.hindawi.com
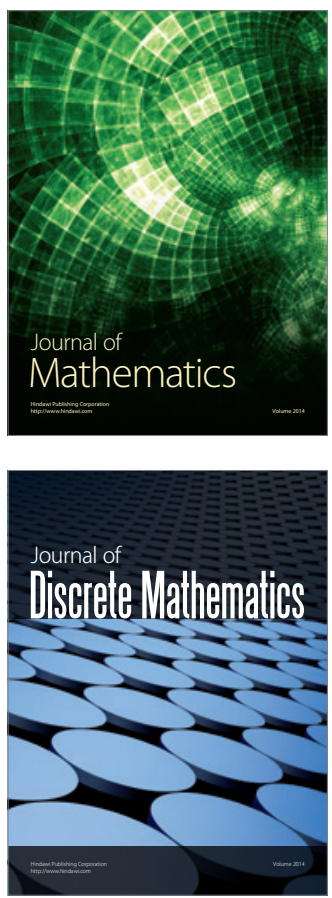

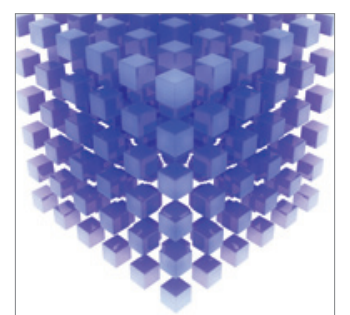

Mathematical Problems in Engineering
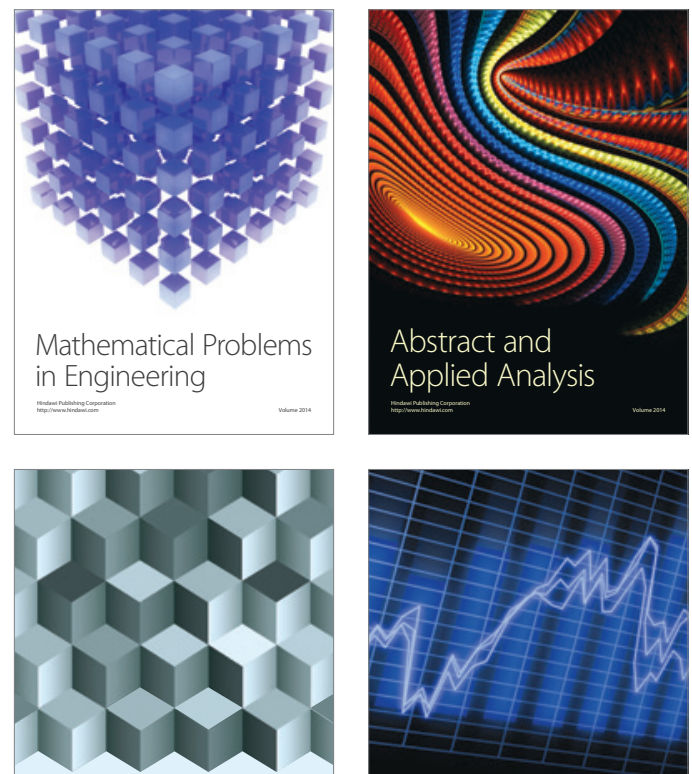

Journal of

Function Spaces

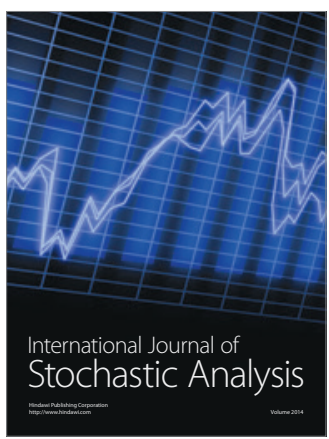

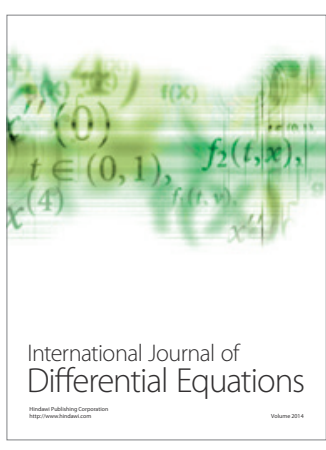
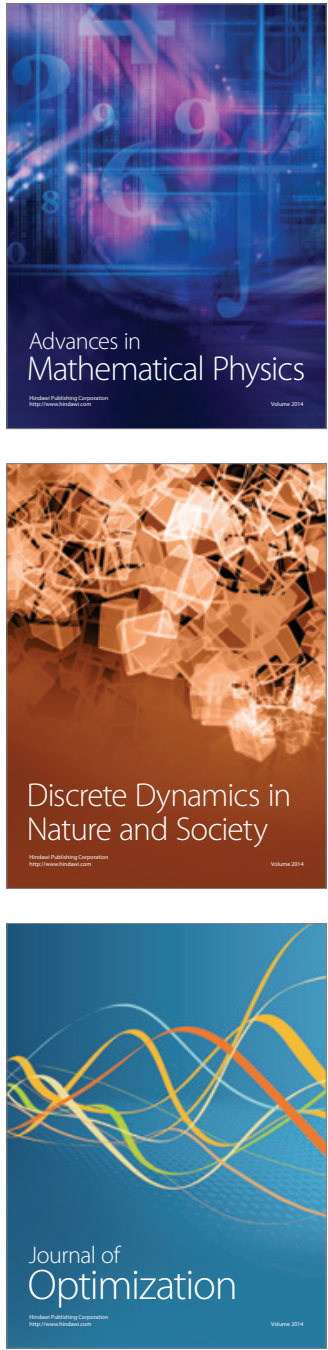